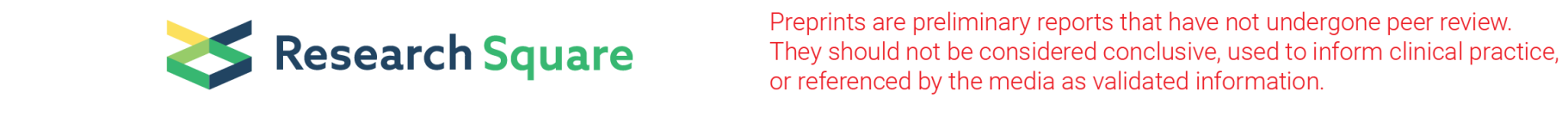

\title{
Tool Condition Monitoring in Milling Process Based on Multi- Dource Pattern Recognition Model
}

\author{
Kui Liang \\ Beihang University \\ Wei Dai \\ Beihang University \\ Tingting Huang ( $\square$ htt@buaa.edu.cn ) \\ Beihang University \\ Zhiyuan Lu \\ Beijing Hangxing Machinery Manufacturing Co., Ltd
}

\section{Research Article}

Keywords: Condition monitoring, Multi-source pattern recognition, Milling processing, Tool wear, Pattern transfer path

Posted Date: March 23rd, 2021

DOI: https://doi.org/10.21203/rs.3.rs-332412/v1

License: (9) (i) This work is licensed under a Creative Commons Attribution 4.0 International License. Read Full License 


\section{Abstract}

In the milling process of metallic parts, appropriate tool condition is essential to reducing processing faults and ensuring manufacturing quality. However, the existing condition monitoring methods are usually limited by recognizing intermediate abnormal states in milling processing, which is inefficient and impractical for real practical applications. Therefore, this paper proposes a Tool condition monitoring (TCM) method in milling process based on multi-source pattern recognition and state transfer path. Firstly, improved K-Means clustering method is used to generate multiple patterns of tool wear. Secondly, a multi-source pattern recognition model framework is developed, and the multiple observation windows and the pattern transfer path are considered in multi-source pattern recognition model. Lastly, PHM2010 datasets are used to verify the feasibility of the proposed method, and the results demonstrate the applicability of the proposed method in practice for tool condition monitoring.

\section{Introduction}

In recent years, the profound changes that have occurred in the field of industrial Internet of Things (IloT), which provided a feasible and convenient service for the development of intelligent manufacturing[1,2]. But the manufacturing process becomes more and more complexed and has brought challenges to our existing monitoring methods at the same time[3,1]. In the field of intelligent manufacturing, various unstable factors and errors in the milling process will not only affect automated production, but also cause economic losses and waste a lot of auxiliary time [4]. In the milling process, the tool directly contacts the workpiece. Tool wear will increase the surface roughness of the workpiece and reduce the quality of the workpiece.[5]Severe tool wear can cause chipping, cracking and chattering, which can damage the workpiece and machine tool, so that lead to serious processing fault [6].

Therefore, TCM methods are essential to reduce processing fault and ensure manufacturing quality[7]. The most primitive TCM method is that the operator estimates the process condition through processing noise, chip shape or cutting vibration differences. This method is entirely dependent on the operator's own experience, which is inefficient and difficult to meet the requirements of complex processes [8]. As for the manufacturing process, many TCM methods have been proposed and developed in recent years. For example, part of the work tried to monitor the condition by establishing physical model[ 9,10$]$. But for the machining process, the physical model is often complicated. Another work started from the idea of image recognition [11,12], which is popular in recent years. The image of the tool is captured, and then analyzing the tool wear state based on digital image processing [13,14]. However, the monitoring accuracy of this method is easily affected by light and physical monitoring angles. Now more popular works are carried out based on signal processing and data mining.[15,16] On the one hand, it benefits from previous research, scholars have studied a variety of signals that can reflect condition changes in tool wear process [17-19]. On the other hand, the tool wear state recognition method based on signal data has been proposed gradually [20-24].

The above methods have made great progress compared with the original method which entire relying on operator. However, they may still have some minor shortcomings when dealing with multi-source data. [25] Data is extremely important for this method and because of the complementation of different signals a huge amount of multi-source data often is collected by various sensors to ensure the monitoring accuracy of tool wear condition, but it's easy to cause data redundancy.[26-28]. Although accuracy can be guaranteed, the massive data put a burden on the calculate cost for equipment.[29] Actually, for tool wear monitoring, we can meet the need for milling process only by obtaining its approximately wear level rather than precise wear value in the actual industrial manufacturing. Therefore, in order to solve the above problems, the pattern recognition has introduced into TCM field[24]. A common way is the control chart pattern recognition (CCPR) [30]. Based on the idea of statistical process control (SPC) [31,32], the fault pattern is established by the typical fault graphs which appeared in time sequence signals. [33] This method generally through classification algorithm to divide the real-time state into specific pattern, then recognize the fault pattern to obtain the tool condition. The CCPR saves computing power and reduces equipment dependence for milling process[34].Moreover, the work of Lu et al. [35] further supplemented the influence effect of the monitoring window for traditional CCPR method, but his work also less considers the stage characteristic of the process, the same pattern at different stages may have different background meanings.

For the stage characteristic of the process, there are some works consider the natural characteristic of the monitored object itself and divide its process into multiple stages or levels for monitoring [36-38]. However, in the aspect of real-time abnormal state monitoring, the existing methods of abnormal state monitoring almost based on constructing fixed threshold. [29] This method has a large deficiency in recognizing intermediate abnormal states and sudden fault in milling processing, which cannot recognize abnormal states in advance. So are less able to advance to configure and optimize the condition monitoring resource of process.

Page $2 / 22$ 
Therefore, it is of great practical significance to construct TCM method that is more suitable for intelligent manufacturing and improving the efficiency and effect of future enterprise condition monitoring. Cheng et al.[39] used the shapelets to construct the pattern, and studied the pattern transfer path to distinguish between normal and abnormal electricity users. This method can reflect the intermediate state and provide us with effective ideas to solve the above problems, but the relevant research has not been applied to TCM field. Based on previous work, this paper proposes a condition monitoring method for tool in milling process using multi-source pattern recognition model. Firstly, improved K-means clustering method is used to generate multiple patterns of tool wear, Secondly, a multi-source pattern recognition model framework is developed, and the multiple observation windows and the pattern transfer path are considered in multi-source pattern recognition model. One kind of efficient method is provided to recognize fault patterns in milling process.

The advantage of this method includes 3 parts at least. (1) The calculate cost is reduced by monitoring patterns of tool wear instead of accurately wear value. (2) This method considered multiple observation windows and multi-source data fusion, which improved accuracy and timeliness for multi-source pattern recognition model. (3) At the same time, this model evolute the patterns by the patterns transfer path, which considered the whole process instead of only single pattern recognition result, this way considered the stage characteristic of the process and make up for the shortcomings of traditional methods that based on fixed threshold in dealing with recognizing intermediate abnormal states and sudden fault. It's a more reasonable way to guide operators to take targeted measures to avoid tool abnormal conditions.

The rest of this article is organized as follows: Section 2 introduces the research methodology for proposed monitoring method of the milling process. Section 3 provides the implementation and validation of the proposed approach through the actual and simulation data of PHM2010 datasets. The experimental results are analyzed and discussed in Section 4. Finally, we present the conclusions of our work in Section 5.

\section{The Multi-source Pattern Recognition Model}

\subsection{Sensitive observation window sizes selection}

For the multi-source pattern recognition model proposed in the paper. Firstly, we need to generate wear pattern. There are many ways can obtain the pattern to match the requirement of the model, one kind of them is cluster. And K-means clustering is the most common and effective clustering algorithm[40], which is suitable to obtain wear pattern in this paper. But the initialization (randomly choosing $\mathrm{K}$ data points in the dataset and dividing the data points randomly into $\mathrm{K}$ subsets) is uncertain for $\mathrm{K}$-means clustering, which cannot guarantee the stability of performance. Particle swarm optimization (PSO) is a population-based stochastic approach for solving continuous and discrete optimization problems[41], which is quite suitable for solving the above problem. Therefore, PSO is used to improve initialization efficiency of $\mathrm{K}$-means clustering and solve the uncertain problem. The tool historical wear data is divided into multiple subsets, which corresponding to different tool condition by the improved K-Means clustering, and each subset represents a pattern. In this way, the multi-source pattern can be obtained. Then we can monitor the multi-source pattern to reflect the tool condition change in the industrial manufacturing process, and the distinguish of pattern can be achieved by using classification algorithm like SVM in the model. [42] Because of the single SVM can only be used to deal with two classification problems, in order to meet the needs of multiple wear patterns, multiple SVM need to be coordinated[43]. During training, the input of SVM is feature $F$, and the output is wearing pattern $M$, we can identify the pattern $M$ based on the input features $F$. some other commonly classifiers also can be utilized for classification, such as the decision tree, acritical neural network, and so on.

On the other hand, in real-time condition monitoring, the work of Lu et al. [35] showed that different wear patterns may have different sensitivity to different sampling windows, that is to say the recognition rate under different windows is different. So, it is necessary to use classification models to test the recognition ability under different observation windows, and to get the best window size for each pattern. The whole process is shown in the Fig. 1 , the window size is $W$, and the window movement stride is $S(S=1,2,3 \ldots W)$. After finishing the test under $W_{i}$ then set $W_{i}=W_{i}+W_{k}$ and perform the test again ( $W_{k}$ is the increment of window size for each time). The classification model is used to test the recognition ability under different observation window $W_{i}$ until it reaches the maximum value (the maximum value cannot be greater than the data size). For example, suppose that one signal sample of 1000 numbers are recognized, and the window movement stride $S$ is 1 , so when $W_{i}=10$, the sample will be recognized 991 times by the classification model. When $W_{i}=20$, it will be recognized 981 times, and so on, until $W_{i}$ reaches the maximum value. At this moment, the 
classification models already have been tested under observation windows of all sizes and the appearance ratio $R$ of each pattern can be calculated for each size observation window. Then we can select the observation windows that corresponding to the highest appearance ratio $R$ as the sensitive window for each pattern.

\subsection{The real-time identification of multi-source pattern}

After the sensitive window of each pattern is determined, then we can use the pattern recognition model to identify the real-time tool wear state. For the real-time collected multi-source signal $L$, each of which has $n$ numbers as input features vector. And there are $m$ observation windows of different sizes, representing $m$ different characteristic of tool conditions. During condition monitoring, the wearing pattern $M$ of the sample data can be calculated based on the classification model of each type of signal with the sliding of each observation window and the outputs of each the classification model are concatenated as a pattern output vector, which the dimension is determined by the maximum observation window size. For example, as shown in Fig. 2 , if there are three observation windows, and their observation window sizes are 2, 4, 6 respectively (i.e., The amount of data processed is 2, 4, 6). Assuming that one signal sample of 100 numbers to be recognized, and the movement stride $S$ of the observation window is 1 . there are 5 types of wearing pattern $M\left(M_{1}, M_{2}, \ldots, M_{5}\right)$ will be

calculated simultaneously under three kinds of observation windows based on the classification model. Thus, three 95-dimensional pattern outputs vectors can be obtained. Then, the probability of each wearing pattern $M$ can be calculated to obtain the probability vector. In order to maintain the monitoring accuracy, it is not common to analyze only one single signal but more to deal with multisource signals in the actual industrial process. The multi-source signals can be handled in the similar way, and the multi-source pattern recognition model framework is constructed, which is shown in Fig. 3. The framework includes two stages, which are multisignal fusion state recognition and concatenation fusion judgment. For the first stage, the multi-source pattern recognition model can be used to recognize signals in various sensitive windows to obtain an $h$-dimensional pattern outputs vector ( $h$ depends on the largest size observation window). Then the probability of each wearing pattern $M$ can be calculated to obtain the g-dimensional probability vector, and $g$ is the number of possible wearing pattern $M$ (for 5 types of wearing pattern $M$ in Fig. $2, g$ is 5 ). Finally, all probability vectors will be concatenated to obtain a $q$-dimensional vector, which as an input vector of classifier of second stage, and the output of classifier is the current pattern. In this way, we can judge the tool condition during this period based on recognition result.

\subsection{The establishment of the pattern transition path diagram}

Based on previous research and actual industrial process, we can know that the tool wear process can be roughly divided into three stages, which are initial wear, normal wear and severe wear stage.[44] As shown as in Fig. 4, we can see that the tool wear increases rapidly at initial wear stage. Because the new tool only has a small area contact with the workpiece surface directly, which caused relatively stress concentration and lead to the narrow surface is quickly ground on the flank surface. After a period of use, the tool surface becomes smooth and stress decreased, then the tool wear reaches the second stage, which is normal wear stage. In this period, tool can be used effectively with stable wear rate. And in the severe wear stage, the tool wear severely, which will lead to the roughness of the workpiece surface increases quickly. However, this will result that the cutting force and cutting temperature increase sharply in turn. So, the tool wear increases sharply again, this is the reason why the tool wear curve appears as shown in Fig. 4.

For the wear process, through the recognition of the wear pattern $M$, we can get the current tool wear level, but lack the ability to recognize the tool condition change in the process for the overall grasp of the abnormal wear state. For example, as shown in Fig. $\mathbf{5}$, the red curve represents abnormal tool wear process and the blue curve represent normal tool wear process. Form Fig. 5, we can find that the normal wear tends to be more in line with the characteristics of the typical wear curve with three-stage, which shown in Fig. 4. But the abnormal tool wear process often does not conform to this process, and the tool may wear quickly and reach severe wear condition at some intermediate stage during abnormal tool wear process.

Therefore, if we only use the tool wear value as the recognition pattern, and then put it into the multi-source pattern recognition model. We can only get the tool wear level, but lack the judgment of the wear intermediate process, so we consider setting other 
pattern from the perspective of wear increment $N$. And using the method that mentioned above to divide the wear increment into $n$ categories, then set them as added patterns which named $N=\left\{N_{1}, N_{2}, \ldots, N_{n}\right\}$, these different patterns represent different wear increment states. As shown as in Fig. 6, the same pattern at different stages may have different background meanings because of the stage characteristic of the process. The wear increment of the tool is the same at point $(a, b, c, d)$ in Fig. 6 , but its corresponding different tool state. Then combined pattern $N$ with pattern $M$ that based on tool wear value, we can match each intermediate segment of tool wear process by $N$ and $M$ and solve the problem mentioned above. Therefore, it becomes easy to distinguish between abnormal and normal conditions for tool wear process. Then the new pattern is established by combining $N$ with $M$, and record it as $V$. the possible combinations are shown in Table 1. Each $V_{i}$ represents one kind of combination for $M$ and $N$, and there is no doubt that normal and abnormal tool wear process have different transition paths. Therefore, comparing the difference of path can distinguish the normal and abnormal process. At the same time, the different monitoring strategies can be adjusted according to the recognized state of $V$, which can help us to perform better in condition monitoring for the tool wear process.

Table 1 the possible combinations between $M$ and $N$

\begin{tabular}{|llllllll|}
\hline$V_{m n}(m, n)$ & $M_{1}$ & $M_{2}$ & $M_{3}$ & $\ldots$ & $M_{5}$ & $M_{6}$ & $M_{m}$ \\
\hline$N_{1}$ & $\left(M_{1}, N_{1}\right)$ & $\left(M_{2}, N_{1}\right)$ & $\left(M_{3}, N_{1}\right)$ & $\ldots$ & $\left(M_{5}, N_{1}\right)$ & $\left(M_{6}, N_{1}\right)$ & $\left(M_{m}, N_{1}\right)$ \\
\hline$N_{2}$ & $\left(M_{1}, N_{2}\right)$ & $\left(M_{2}, N_{2}\right)$ & $\left(M_{3}, N_{2}\right)$ & $\ldots$ & $\left(M_{5}, N_{2}\right)$ & $\left(M_{6}, N_{2}\right)$ & $\left(M_{m}, N_{2}\right)$ \\
\hline$N_{3}$ & $\left(M_{1}, N_{3}\right)$ & $\left(M_{2}, N_{3}\right)$ & $\left(M_{3}, N_{3}\right)$ & $\ldots$ & $\left(M_{5}, N_{3}\right)$ & $\left(M_{6}, N_{3}\right)$ & $\left(M_{m}, N_{3}\right)$ \\
$\ldots$ & $\ldots$ & $\ldots$ & $\ldots$ & $\ldots$ & $\ldots$ & $\ldots$ & $\ldots$ \\
$N_{5}$ & $\left(M_{1}, N_{5}\right)$ & $\left(M_{2}, N_{5}\right)$ & $\left(M_{3}, N_{5}\right)$ & $\ldots$ & $\left(M_{5}, N_{5}\right)$ & $\left(M_{6}, N_{5}\right)$ & $\left(M_{m}, N_{5}\right)$ \\
$N_{6}$ & $\left(M_{1}, N_{6}\right)$ & $\left(M_{2}, N_{6}\right)$ & $\left(M_{3}, N_{6}\right)$ & $\ldots$ & $\left(M_{5}, N_{6}\right)$ & $\left(M_{6}, N_{6}\right)$ & $\left(M_{m}, N_{6}\right)$ \\
$N_{n}$ & $\left(M_{1}, N_{n}\right)$ & $\left(M_{2}, N_{n}\right)$ & $\left(M_{3}, N_{n}\right)$ & $\ldots$ & $\left(M_{5}, N_{n}\right)$ & $\left(M_{6}, N_{n}\right)$ & $\left(M_{m}, N_{n}\right)$ \\
\hline
\end{tabular}

\section{Implementation And Validation Of The Proposed Approach}

\subsection{Experiment setup}

To demonstrate the effectiveness of our contributions, the experimental data is measured from a high-speed milling process, which is obtained from "the International PHM Data Challenge Competition in 2010" database.[45] The performance of the proposed method is verified based on that database. A high-speed CNC milling machine is used for milling operation in the experiment, and the workpiece surface processed into an inclined plane of $60^{\circ}$. The experiment structure is shown in Fig. 7, and the tool related parameter information is shown in Table 2.

The experiment used a three-way dynamometer, three-way accelerometers, and an acoustic emission (AE), there are total seven sensing channels for three types of sensors. The experimental installation is shown in Fig. 8. Among them, a Kistler three-way dynamometer is installed between the workpiece and the processing table to measure the cutting force based on electric charge, which is converted into voltage by charge amplifier. Three Kistler piezoelectric accelerometers are installed on the workpiece to measure the vibration of the workpiece in the $X, Y$ and $Z$ directions during the milling process. The $A E$ sensor is installed on the side of the workpiece to monitor the high-frequency acoustic emission signal during the milling process., Meanwhile, the radial cutting is $0.125 \mathrm{~mm}$ and the axial cutting $0.2 \mathrm{~mm}$ with $50 \mathrm{kHz}$ sampling frequency for each completed machining process. After each processing, the Leica MZ 12 microscope will be used to measure the corresponding side surface wear of cutter.

Table 2 PHM2010 competition experiment parameter list 


\begin{tabular}{|llll|}
\hline Parameter & value & parameter & value \\
\hline Machine model & Roders Tech RFM 760 & Radial depth of cut & $0.125 \mathrm{~mm}$ \\
\hline Workpiece material & Nickel-based superalloy 718 & axial cutting depth & $0.2 \mathrm{~mm}$ \\
\hline Tool & 3-tooth ball nose milling cutter & Number of sensors & 3 \\
\hline Spindle speed & 10,400 RPM & Number of sensing channels & 7 \\
\hline Feed rate & $1555 \mathrm{~mm} / \mathrm{min}$ & Sampling frequency & $50 \mathrm{kHz}$ \\
\hline
\end{tabular}

\subsection{Data processing and pattern generation}

According to the actual operating conditions of the manufacturing process, the corresponding multi-source sensors are arranged reasonably. Then the multi-source data is collected and preprocessed by arranged sensors. Firstly, we perform data cleaning and noise reduction on the signal data to exclude irrelevant information interference for results. Secondly, the processed data is conducted to feature extraction and feature selection to obtain a suitable feature. This will help us eliminate information interference further and improve accuracy[46]. Lastly, the historical tool wear data is used to generate wear pattern, which different patterns represent different tool states during tool wear process.

The original data often contains noise and other irrelevant information that will interfere with our analysis results, so the data which in PHM2010 dataset need to be preprocessed. Here the wavelet threshold method was choosing to noise reduction for the data collected by the 7 sensor channels. the 5 layers haar wavelet was used in the model with the heuristic threshold, which could adjust the threshold according to the noise decomposed in the first wavelet layer. Then the feature extraction on the processed data was conducted, and extracted 17 time-domain features, 4 frequency-domain features, and some time-frequency domain features. Among them, 17 time-domain features included common dimensional and non-dimensional indicators. The 4 frequency-domain features were gravity frequency, average frequency, root mean square (RMS) frequency and standard deviation of frequency. And the timefrequency domain features were scale entropy, energy entropy, singular entropy of wavelet. The partially extracted features were shown in the Fig. 9.Then normalized them and calculated the Pearson correlation coefficient according to the tool wear value. Further we performed feature selection, and then the PCA (Principal Component Analysis) was used to dimensional reduction with correlation criterion 0.85 . The contribution rate was set to be greater than $95 \%$, and the principal components were sorted by variance to get the final feature set which was recorded as $F$.

And according to the stage characteristic of tool as mentioned in Section 2, the wear value can be divided into three types of pattern. But the number of patterns need to be adjusted appropriately to meet actual needs. To consider with the appropriate accuracy of monitoring, based on improved K-means clustering algorithm, the wear data of PHM2010 dataset was divided into 5 categories and set each category as one type of wear pattern $M=\left\{M_{1}, M_{2}, \ldots, M_{5}\right\}$. And comparing with the wear value that measured by microscope, we could obtain the corresponding relationship between wear level and pattern, which was shown in Fig. 10. Each pattern $M$ corresponds to a segment of the wear curve, and $M_{1}$ was the initial wear pattern, $M_{5}$ represent the most severe wear state. From $M_{1}$ to $M_{5}$, the severity of tool wear increased continually, so we could replace the tool when it be recognized as $M_{4}$ or $M_{5}$ by the model that build in section 2 in actual milling process, to ensure the quality and efficiency of the process. Although this method could not obtain accurate tool wear values, it was sufficient for general manufacturing processes.

As mentioned in section 2, to monitor the wear intermediate process, we need to generate the wear increment pattern, and the same method was used to divide the wear increment into 5 categories and set each category as one type of pattern $N=\left\{N_{1}, N_{2}, \ldots, N_{5}\right\}$. the corresponding relationship between wear increment categories and the patterns was shown in Fig. 11. And these different patterns represented different wear increment states. Finally, combined pattern $N$ with pattern $M$ to obtain the new pattern $V$ which were shown in Table 1, and put pattern $V$ into the multi-source pattern recognition model to carry out the condition monitoring for the tool wear process.

\subsection{Validation of the proposed approach}

Page 6/22 
Before the multi-source pattern recognition model was used, which mentioned in Section 2 as shown in Fig. 2 and Fig. 3, we need to confirm the sensitive window size of each pattern, our model was used to recognize all sample under different size of observation windows and calculate the proportion of each pattern in different observation windows. Take the severe wear pattern recognition as an example, one signal sample of 12,700 data points were recognized, and the window movement stride $S$ were 1 , the result is shown in Fig. 12. From Fig. 12, we can find that the proportion of pattern $M_{5}$ is the largest with the observation window size near 2500. Although the recognition proportion increase with the window size later, the smaller the window means better timeliness. Therefore, we set the sensitive window of pattern $M_{5}$ to 2500 is more reasonable. Similarly, the above steps can be repeated until all sensitive windows for each pattern are obtained. lastly, for the condition monitoring of the milling process, it is only necessary to use the established multi-source pattern recognition model to monitor and recognize tool pattern under these sensitive observation windows.

The next step is to obtain the pattern transition path. The multi-source data of milling process can be recognized by the multi-source pattern recognition model under different observation window, and we can get its recognized pattern in real time. With the recognition of wear patterns $M$ that based on tool wear value, we can get the wear level of tool. And we can replace the tool when it be recognized as $M_{4}$ or $M_{5}$ which indicate terrible condition for tool to ensure the quality and efficiency of the process. As mentioned above, we can only obtain wear level by monitoring patterns $M$, and the process cannot be judged whether its normal or abnormal. Therefore, the wear incremental pattern $N$ is constructed according to the same idea, and the pattern $V(M, N)$ was constructed by combining the $M$ and $N$. In this way, we can match each intermediate segment of tool wear process. Each $V_{i}$ represents one kind of combination for $M$ and $N$. Then we set the original PHM 2010 datasets as the normal wear process group and get the abnormal process group by simulating based on the process. Finally, we experiment with the proposed model in these two groups and obtained the pattern transition path. Tool wear pattern transfer path diagram is presented in Fig. 13, illustrating how wear pattern would transfer from one to another in the milling process. In Fig. 13, The node is pattern $V_{i}$, each $V_{i}$ represents one kind of combination for $M$ and $N$. For each node, each vertex size is proportional to its weighted degree, and the width of the edge is proportional to its betweenness. At the same time, according to Fig. 13,. for the normal tool wear record, there is a clear path for its pattern transition " $V_{7}$ à $V_{5}$ à $V_{15}$ à $V_{22}$ " in the graph. For the abnormal data, however, the path " $V_{7}$ à $V_{5}$ à $V_{15}$ à $V_{22}$ " does not exist and more in line with path " $V_{1}$ à $V_{6}$ à $V_{17}$ à $V_{22}$ ", indicating that the connectivity of the pattern transition path provides an evidential basis for detecting an abnormal wear process. According to

the idea of working with Cheng et al.[39], we can compare the paths changes to judge the tool wear process is normal wear or not.

Next, the recognition ability of proposed method is tested by C4 in PHM 2010 dataset. And the C4 is divided into two subsets, one is the training subset which named $T_{1}$, and the other is the test subset which named $T_{2}$. Firstly, $T_{1}$ is used to train model according to the method in the Section 2. There are 315 groups in C4, then the first 5 groups are regarded as unworn state and the last 5 groups are regarded as severe wear state. Considering the complexity of the condition monitoring in this case study, the effectiveness of signal needs to be evaluated. Take the cutting force signal in $\mathrm{X}$ dimension as an example, the difference of tool signal between unworn state and severe wear state is shown in the Fig. 14. It is noticed that the two states have a very obvious difference, which is the signal amplitude of the unworn state is much smaller than severe wear state. It is clear that the cutting force signal can reflect tool wear condition with high sensitivity, which demonstrates the effectiveness of this type of signal for tool wear monitoring in this study. Other signals can also be analyzed in a similar way to be evaluated for tool wear monitoring.

To evaluate the performance of the proposed method for the current case study. And the training model is used to recognize $T_{2}$ and the result as shown in Fig. 15. It is evident that the signal amplitude of cutting force has shown significant variation with respect to the reference tool wear from unworn to severe wear state, and the change point of wear pattern for the tool also is displayed in Fig. 15 (a). In addition, it has been noticed that the wear pattern before $K_{1}$ is detected as $M_{1}$ and the pattern after $K_{2}$ is recognized as $M_{5}$. In order to verify the effectiveness of recognition result, the actual wear value is compared as shown in Fig. $\mathbf{1 5}$ (b), it is clear that the points $K_{1}$ and $K_{2}$ of actual wear value are consistent with the recognized pattern. This observed result clearly shows the performance of proposed monitoring approach to detect the wear development trend in this case study, which proves that the approach has the ability to recognize tool wear state.

Furthermore, all the measured signal data of $\mathrm{C} 4$ is used to evaluate the performance of the proposed approach to distinguish normal and abnormal process in this case study. The multi-source data of $\mathrm{C} 4$ is recognized by the multi-source pattern recognition model 
under different observation window, and the recognized pattern is obtained in real time. The recognized pattern including two parts, which are wear patterns $M$ and the wear incremental pattern $N$. As mentioned above, the pattern transfer path can be obtained by combining the $M$ and $N$ during the monitoring process. And the pattern transfer path of $C 4$ is " $V_{7}$ à $V_{5}$ à $V_{15}$ à $V_{22}$ ". It is noticed that the pattern transfer path of $\mathrm{C} 4$ consistent with the pattern transfer path of normal process, which is shown in Fig. 13. Therefore, this observed result clearly shows the performance of proposed monitoring approach to detect the wear normal and abnormal process, which proves that the approach is generally feasible to distinguish tool wear state.

\section{Comparison And Discussion}

\subsection{Comparison of methods}

In addition, other condition monitoring methods (including KNN, ANN, SVM, and decision tree) were used to detect abnormal processing conditions based on the same datasets. The experiment was carried out in three kind of ways which were single channel signal (cutting force $(\mathrm{N})$ in $\mathrm{X}$ dimension signals), fixed observation window and multi-channel variable window. Then, the common time-domain features, frequency-domain features and time-frequency do-main features of each sample that from PHM2010 were extracted, and the sensitive features related to abnormal machining condition were selected as the input of these model. For single channel signal, the cutting force signals data in $\mathrm{X}$ dimensions were recognized by the model under different observation window as shown as in Fig. 2, the cutting force signals in $X$ dimensions were integrated in this case, and the inputs were 200 mean values for every second (one mean value represents 250 raw data with $50 \mathrm{kHz}$ sampling frequency). The stride was set as 2 , which means that the 500 newly collected data can be used to calculate 100 newly recognition results of sensor by model, the Multi-class SVMs were trained and the validation method was the cross-validation method. Then the proportion of each pattern was calculated to deicide the pattern. In fixed observation window way, the influence effect of the observed window was not considered in the proposed method. And in multi-channel variable window way, the multi-source data were recognized by the multi-source pattern recognition model under different observation window as shown as in Fig. 3. and according to Fig. 3, all probability vectors will be concatenated as the input of the second stage classifier to obtain finial result. And then evaluated the monitoring performance of KNN, ANN, SVM and decision tree with the detection accuracy of wear state which is to judge the tool wear pattern, and processing condition which is to judge whether the process is abnormal or not. All the result is shown in Table 3 . It is noticed that the ability to recognize the wear state and the processing condition is different. The best perform of the proposed model was observed in Multi-channel variable window way and under a fixed observation window achieved the worst result. the recognition efficiency of single channel signal only slightly better than the fixed window way. This observed result clearly shown the proposed monitoring approach obtained the best recognition accuracy under multi-channel variable window and using SVM for classification to detect the wear development trend in this case study, which proved that considering the multi-source data and variable observation window for different pattern improved the monitoring ability significantly.

Table 3 Performance of proposed method under the different condition

\begin{tabular}{|llllllllll|}
\hline \multicolumn{3}{|l}{ Recognition accuracy } & & & & & & \\
& \multicolumn{2}{l}{$\begin{array}{l}\text { Single channel signal }(X \text { force } \\
\text { signal) }\end{array}$} & Fixed recognition window & & \multicolumn{2}{l|}{$\begin{array}{l}\text { Multi-channel variable } \\
\text { window }\end{array}$} \\
\hline Classifier & $\begin{array}{l}\text { Wear } \\
\text { condition }\end{array}$ & $\begin{array}{l}\text { Processing } \\
\text { condition }\end{array}$ & $\begin{array}{l}\text { Average } \\
\text { accuracy }\end{array}$ & $\begin{array}{l}\text { Wear } \\
\text { condition }\end{array}$ & $\begin{array}{l}\text { Processing } \\
\text { condition }\end{array}$ & $\begin{array}{l}\text { Average } \\
\text { accuracy }\end{array}$ & $\begin{array}{l}\text { Wear } \\
\text { condition }\end{array}$ & $\begin{array}{l}\text { Processing } \\
\text { condition }\end{array}$ & $\begin{array}{l}\text { Average } \\
\text { accuracy }\end{array}$ \\
\hline TREE & $88.6 \%$ & $84.7 \%$ & $86.7 \%$ & $84.5 \%$ & $80.8 \%$ & $82.7 \%$ & $91.1 \%$ & $90.4 \%$ & $90.8 \%$ \\
\hline ANN & $87.9 \%$ & $83.1 \%$ & $85.5 \%$ & $88.1 \%$ & $82.1 \%$ & $85.1 \%$ & $92.9 \%$ & $91.4 \%$ & $92.2 \%$ \\
\hline KNN & $90.5 \%$ & $76.8 \%$ & $83.7 \%$ & $85.5 \%$ & $79.8 \%$ & $82.7 \%$ & $90.5 \%$ & $88.7 \%$ & $89.6 \%$ \\
\hline SVM & $91.20 \%$ & $83.40 \%$ & $87.3 \%$ & $87.50 \%$ & $83.60 \%$ & $85.6 \%$ & $93.60 \%$ & $92.60 \%$ & $93.1 \%$ \\
\hline
\end{tabular}

\subsection{Discussion}


The PHM2010 dataset was used to validate the feasibility of proposed method by recognizing tool wear condition. Compared with the existing condition monitoring methods. The proposed method benefits from the ability to fuse multi-sensor high frequency data and takes into account the influence of different observation windows for each pattern, which improved the effectiveness of monitoring and recognition accuracy greatly. In addition, the proposed method also considered to evolute the recognized patterns form the pattern transfer path, not only the results of pattern recognition. The abnormal conditions are recognized by fault transfer path in the proposed methodology, and compared with the traditional method that by fixed threshold, the proposed methodology directly reflect abnormal conditions to quickly find the cause of the abnormality. And it make up for the shortcomings in dealing with intermediate states $₫$ which can more reasonably monitoring tool condition and guide the user to take corrective actions to prevent poor end product quality. Thus, the proposed method is one kind of efficient way to detect the abnormal tool conditions based on the high frequency online data of multi-sensor during the milling process. In order to improve the effectiveness of the proposed method in practical applications, the following basic issues must be considered to continue to expand and improve the method:

(1) The method proposed in this paper only considers the recognition of tool wear. However, in actual manufacturing applications, some other abnormal conditions may occur at the same time, such as chattering, tool looseness, etc. Therefore, this method should further consider the difference of wear development trend under different abnormal condition and try to distinguish the type of abnormal condition.

(2) The different patterns are the key for detecting tool wear condition in this paper. However, the principle of pattern generation mainly considers the changes of various signal data and the division of the mode mainly according to the tool wear condition. Therefore, when working condition changed, the patterns are easily misidentified, so that the parameters related to these patterns should be adjusted according to the real working scenes.

\section{Conclusions}

In order to solve the problem of abnormal condition monitoring during the milling process, this paper proposes a tool condition monitoring method for milling process based on using multi-source pattern recognition model and fault transfer path. The improved K-means clustering method is used to generate multiple patterns of tool wear at first. Secondly, a multi-source data fusion model framework is developed, and the multiple observation windows and the pattern transfer path are considered in multi-source pattern recognition model. This paper gives two key contributions: the establishment of a multi-source pattern recognition model and the abnormal judgment based on fault paths. By combining data collected from multiple-sensors and multiple observation windows, an information fusion framework is established to monitor the tool milling process, which can realize tool wear condition recognition and consider the pattern transfer path to distinguish abnormal and normal wear. Lastly, the PHM2010 dataset was used for feasibility verification of the proposed method. The experimental results proved the applicability of the proposed method for tool condition monitoring. However, the current work still needs to be extended to make it more suitable for handling real milling processing scenarios. For example, further consider the difference of wear development trend to distinguish the abnormal condition type, and testing the recognition effect of the model under different working conditions. In addition, other more reasonable way also can be considered to generation patterns for the proposed approach

\section{Declarations}

Ethical Approval: Not applicable.

Consent to Participate: Not applicable.

Consent to Publish: Not applicable.

Funding: The authors acknowledge the financial support of National Natural Science Foundation of China (No.51705015), and National Defense Fundamental Research Foundation of China (No. JCKY2018203C005).

Conflicts of interest: The authors declare that no conflict of interests exits in the submission of this manuscript.

Availability of data and material: The datasets during the current study are available in "PHM Data Challenge 2010" database, https://www.Phmsociety.org/competition/phm/10S. 
Authors' contributions: Kui Liang contributed to the conception, methodology and write original draft for the study. Wei Dai performed supervising, writing-review for the manuscript. Tingting Huang contributed significantly to analysis, review and editing the manuscript. Zhiyuan Lu helped perform the analysis with constructive discussions.

\section{References}

1. P Sunhare, RR Chowdhary, MK Chattopadhyay. (2020). Internet of things and data mining: An application oriented survey. Journal of King Saud University - Computer and Information Sciences. doi: 10.1016/j.jksuci.2020.07.002

2. K Li, T Zhou, B Liu. (2020). Internet-based intelligent and sustainable manufacturing: developments and challenges. The International Journal of Advanced Manufacturing Technology, 108(5-6), 1767-1791. doi: 10.1007/s00170-020-05445-0

3. H Hu, L Wang, P Luh. (2015). Intelligent manufacturing: New advances and challenges. J INTELL MANUF, 26(5), 841-843. doi: $10.1007 / \mathrm{s} 10845-015-1148-\mathrm{z}$

4. H Chelladurai, VK Jain, NS Vyas. (2008). Development of a cutting tool condition monitoring system for high speed turning operation by vibration and strain analysis. The International Journal of Advanced Manufacturing Technology, 37(5-6), 471-485. doi: 10.1007/s00170-007-0986-z

5. K Javed, R Gouriveau, X Li, N Zerhouni. (2018). Tool wear monitoring and prognostics challenges: a comparison of connectionist methods toward an adaptive ensemble model. J INTELL MANUF, 29(8), 1873-1890. doi: 10.1007/s10845-016$1221-2$

6. J Guo, A Li, R Zhang. (2020). Tool condition monitoring in milling process using multifractal detrended fluctuation analysis and support vector machine. The International Journal of Advanced Manufacturing Technology, 110(5-6), 1445-1456. doi: 10.1007/s00170-020-05931-5

7. T Xia, Y Dong, L Xiao, S Du, E Pan, L Xi. (2018). Recent advances in prognostics and health management for advanced manufacturing paradigms. RELIAB ENG SYST SAFE, 178, 255-268. doi: 10.1016/j.ress.2018.06.021

8. T Mohanraj, S Shankar, R Rajasekar, NR Sakthivel, A Pramanik. (2020). Tool condition monitoring techniques in milling process a review. Journal of Materials Research and Technology, 9(1), 1032-1042. doi: 10.1016/j.jmrt.2019.10.031

9. P Goodall, D Pantazis, A West. (2020). A cyber physical system for tool condition monitoring using electrical power and a mechanistic model. COMPUT IND, 118, 103223. doi: 10.1016/j.compind.2020.103223

10. M Nouri, BK Fussell, BL Ziniti, E Linder. (2015). Real-time tool wear monitoring in milling using a cutting condition independent method. International Journal of Machine Tools and Manufacture, 89, 1-13. doi: 10.1016/j.ijmachtools.2014.10.011

11. H Oo, W Wang, Z Liu. (2020). Tool wear monitoring system in belt grinding based on image-processing techniques. The International Journal of Advanced Manufacturing Technology, 111(7-8), 2215-2229. doi: 10.1007/s00170-020-06254-1

12. A Qin, L Guo, Z You, H Gao, X Wu, S Xiang. (2020). Research on automatic monitoring method of face milling cutter wear based on dynamic image sequence. The International Journal of Advanced Manufacturing Technology, 110(11-12), 3365-3376. doi: 10.1007/s00170-020-05955-x

13. L Fernández-Robles, L Sánchez-González, J Díez-González, M Castejón-Limas, H Pérez. (2020). Use of image processing to monitor tool wear in micro milling. NEUROCOMPUTING. doi: 10.1016/j.neucom.2019.12.146

14. NN Bhat, S Dutta, T Vashisth, S Pal, SK Pal, R Sen. (2016). Tool condition monitoring by SVM classification of machined surface images in turning. The International Journal of Advanced Manufacturing Technology, 83(9-12), 1487-1502. doi: $10.1007 / \mathrm{s} 00170-015-7441-3$

15. P Ong, WK Lee, RJH Lau. (2019). Tool condition monitoring in CNC end milling using wavelet neural network based on machine vision. The International Journal of Advanced Manufacturing Technology, 104(1-4), 1369-1379. doi: 10.1007/s00170-01904020-6

16. B Shen, Y Gui, B Chen, Z Lin, Q Liu, Q Liu. (2020). Application of spindle power signals in tool condition monitoring based on HHT algorithm. The International Journal of Advanced Manufacturing Technology, 106(3-4), 1385-1395. doi: 10.1007/s00170019-04684-0

17. M Uekita, Y Takaya. (2017). Tool condition monitoring for form milling of large parts by combining spindle motor current and acoustic emission signals. The International Journal of Advanced Manufacturing Technology, 89(1-4), 65-75. doi: 
18. Z Xie, Y Lu, X Chen. (2020). A multi-sensor integrated smart tool holder for cutting process monitoring. The International Journal of Advanced Manufacturing Technology, 110(3-4), 853-864. doi: 10.1007/s00170-020-05905-7

19. MSH Bhuiyan, IA Choudhury, M Dahari. (2014). Monitoring the tool wear, surface roughness and chip formation occurrences using multiple sensors in turning. J MANUF SYST, 33(4), 476-487. doi: 10.1016/j.jmsy.2014.04.005

20. R Liu, A Kothuru, S Zhang. (2020). Calibration-based tool condition monitoring for repetitive machining operations. J MANUF SYST, 54, 285-293. doi: 10.1016/j.jmsy.2020.01.005

21. M Soualhi, KTP Nguyen, K Medjaher. (2020). Pattern recognition method of fault diagnostics based on a new health indicator for smart manufacturing. MECH SYST SIGNAL PR, 142, 106680. doi: 10.1016/j.ymssp.2020.106680

22. M Lamraoui, M Thomas, M El Badaoui. (2014). Cyclostationarity approach for monitoring chatter and tool wear in high speed milling. MECH SYST SIGNAL PR, 44(1-2), 177-198. doi: 10.1016/j.ymssp.2013.05.001

23. T Mohanraj, S Shankar, R Rajasekar, NR Sakthivel, A Pramanik. (2020). Tool condition monitoring techniques in milling process - a review. Journal of Materials Research and Technology, 9(1), 1032-1042. doi: 10.1016/j.jmrt.2019.10.031

24. F Zorriassatine, A Al-Habaibeh, RM Parkin, MR Jackson, J Coy. (2005). Novelty detection for practical pattern recognition in condition monitoring of multivariate processes: a case study. The International Journal of Advanced Manufacturing Technology, 25(9-10), 954-963. doi: 10.1007/s00170-004-2174-8

25. Z Lu, M Wang, W Dai, J Sun. (2019). In-process complex machining condition monitoring based on deep forest and process information fusion. The International Journal of Advanced Manufacturing Technology, 104(5-8), 1953-1966. doi: 10.1007/s00170-019-03919-4

26. P Stavropoulos, A Papacharalampopoulos, E Vasiliadis, G Chryssolouris. (2016). Tool wear predictability estimation in milling based on multi-sensorial data. The International Journal of Advanced Manufacturing Technology, 82(1-4), 509-521. doi: $10.1007 / \mathrm{s} 00170-015-7317-6$

27. H Wang, G Ni, J Chen, J Qu. (2020). Research on rolling bearing state health monitoring and life prediction based on PCA and Internet of things with multi-sensor. MEASUREMENT, 157, 107657. doi: 10.1016/j.measurement.2020.107657

28. M Rizal, JA Ghani, MZ Nuawi, CHC Haron. (2017). Cutting tool wear classification and detection using multi-sensor signals and Mahalanobis-Taguchi System. WEAR, 376-377, 1759-1765. doi: 10.1016/j.wear.2017.02.017

29. SY Wong, JH Chuah, HJ Yap. (2020). Technical data-driven tool condition monitoring challenges for CNC milling: a review. The International Journal of Advanced Manufacturing Technology, 107(11-12), 4837-4857. doi: 10.1007/s00170-020-05303-z

30. D Fuqua, T Razzaghi. (2020). A cost-sensitive convolution neural network learning for control chart pattern recognition. EXPERT SYST APPL, 150, 113275. doi: 10.1016/j.eswa.2020.113275

31. SW Roberts. (2000). Control chart tests based on geometric moving averages.

32. GB Wetherill. (1977). Cumulative sum charts.

33. A Addeh, A Khormali, NA Golilarz. (2018). Control chart pattern recognition using RBF neural network with new training algorithm and practical features. ISA T, 79, 202-216. doi: 10.1016/j.isatra.2018.04.020

34. A Aziz Kalteh, S Babouei. (2020). Control chart patterns recognition using ANFIS with new training algorithm and intelligent utilization of shape and statistical features. ISA T, 102, 12-22. doi: 10.1016/j.isatra.2019.12.001

35. Z Lu, M Wang, W Dai. (2020). A condition monitoring approach for machining process based on control chart pattern recognition with dynamically-sized observation windows. COMPUT IND ENG, 142, 106360. doi: 10.1016/j.cie.2020.106360

36. Y Jiang, Z Xu, Z Zhang, X Liu. (2019). A novel shearer cutting pattern recognition model with chaotic gravitational search optimization. MEASUREMENT, 144, 225-233. doi: 10.1016/j.measurement.2019.05.019

37. P Lyu, L Yao, X Ma, G An, G Bai, AT Augousti, X Tong. (2020). Correlation between failure mechanism and rupture lifetime of 2D$\mathrm{C} / \mathrm{SiC}$ under stress oxidation condition based on acoustic emission pattern recognition. J EUR CERAM SOC, 40(15), 5094-5102. doi: 10.1016/j.jeurceramsoc.2020.06.070

38. IBM Taha, SS Dessouky, SSM Ghoneim. (2021). Transformer fault types and severity class prediction based on neural patternrecognition techniques. ELECTR POW SYST RES, 191, 106899. doi: 10.1016/j.epsr.2020.106899

39. Z Cheng, Y Yang, W Wang, W Hu, YASG Zhuang. (2020). Time2Graph: Revisiting Time Series Modeling with Dynamic Shapelets. Paper presented at the Association for the Advancement of Artificial Intelligence $\triangle A A A I \mathbb{Z}$ 
40. X Jin, J Han. (2010). K-Means Clustering. In C Sammut, GI Webb (Eds.), Encyclopedia of Machine Learning (563-564). Boston, MA: Springer US. (Reprinted. doi: 10.1007/978-0-387-30164-8_425.

41. K J., E R. (0027-01-19). Particle swarm optimizationProceedings of ICNN'95 - International Conference on Neural Networks, 19951942-1948. doi: 10.1109/ICNN.1995.488968

42. C Cortes, V Vapnik. (1995). Support-Vector Networks. Machine Learning, 1(20), 273-297

43. RG Brereton, GR Lloyd. (2010). Support Vector Machines for classification and regression. The Analyst, 135(2), 230-267. doi: 10.1039/B918972F

44. Y Zhou, W Xue. (2018). Review of tool condition monitoring methods in milling processes. The International Journal of Advanced Manufacturing Technology, 96(5-8), 2509-2523. doi: 10.1007/s00170-018-1768-5

45. PHM Society (2010) PHM Data Challenge. (2010). PHM Society (2010) PHM Data Challenge. Available. URL. https://www. Phmsociety.org/competition/phm/10S

46. Z Xie, J Li, Y Lu. (2019). Feature selection and a method to improve the performance of tool condition monitoring. The International Journal of Advanced Manufacturing Technology, 100(9-12), 3197-3206. doi: 10.1007/s00170-018-2926-5

\section{Figures}

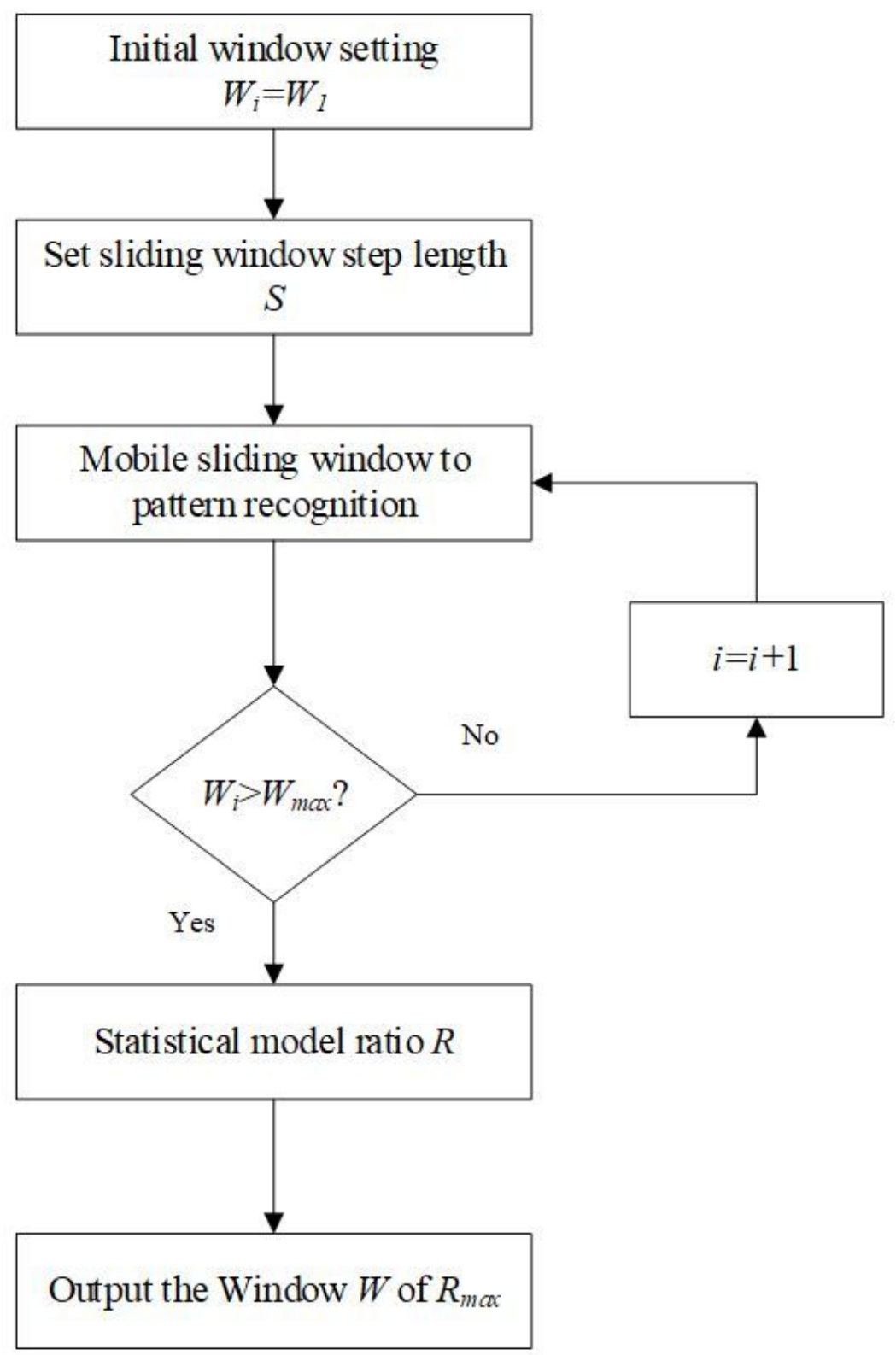




\section{Figure 1}

Sensitive window selection flowchart

\section{Moving sliding window state recognition}

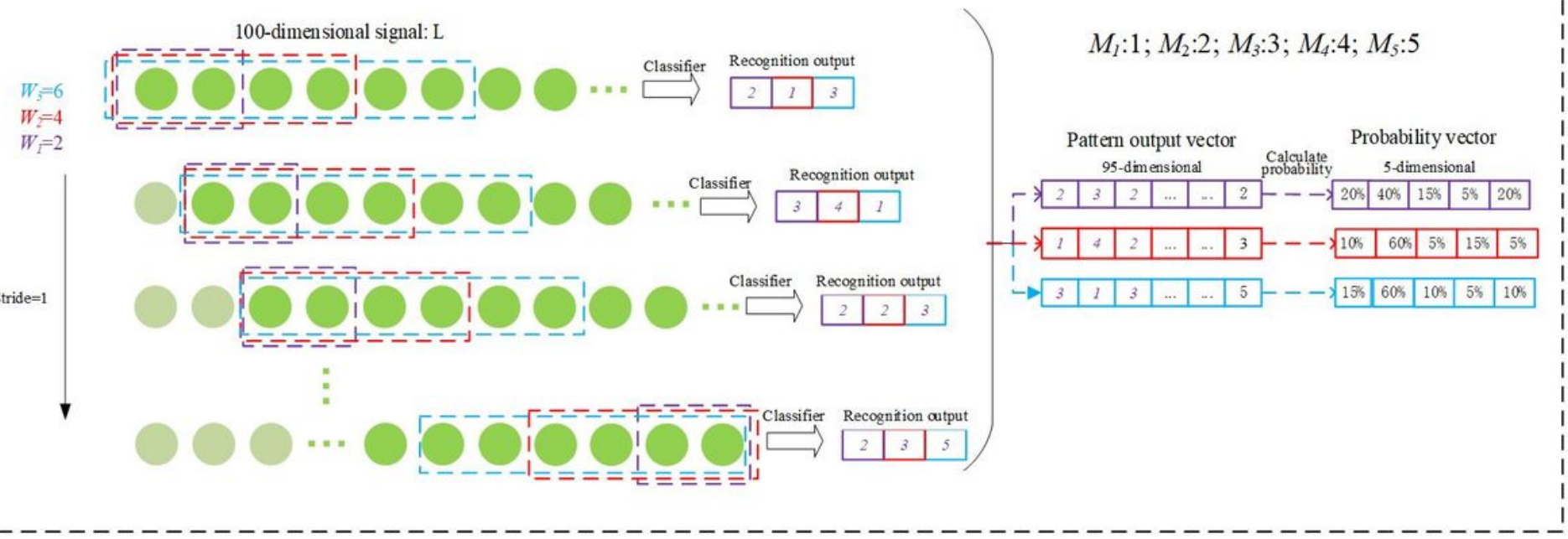

Figure 2

Schematic diagram of state recognition of moving sliding window based on single signal

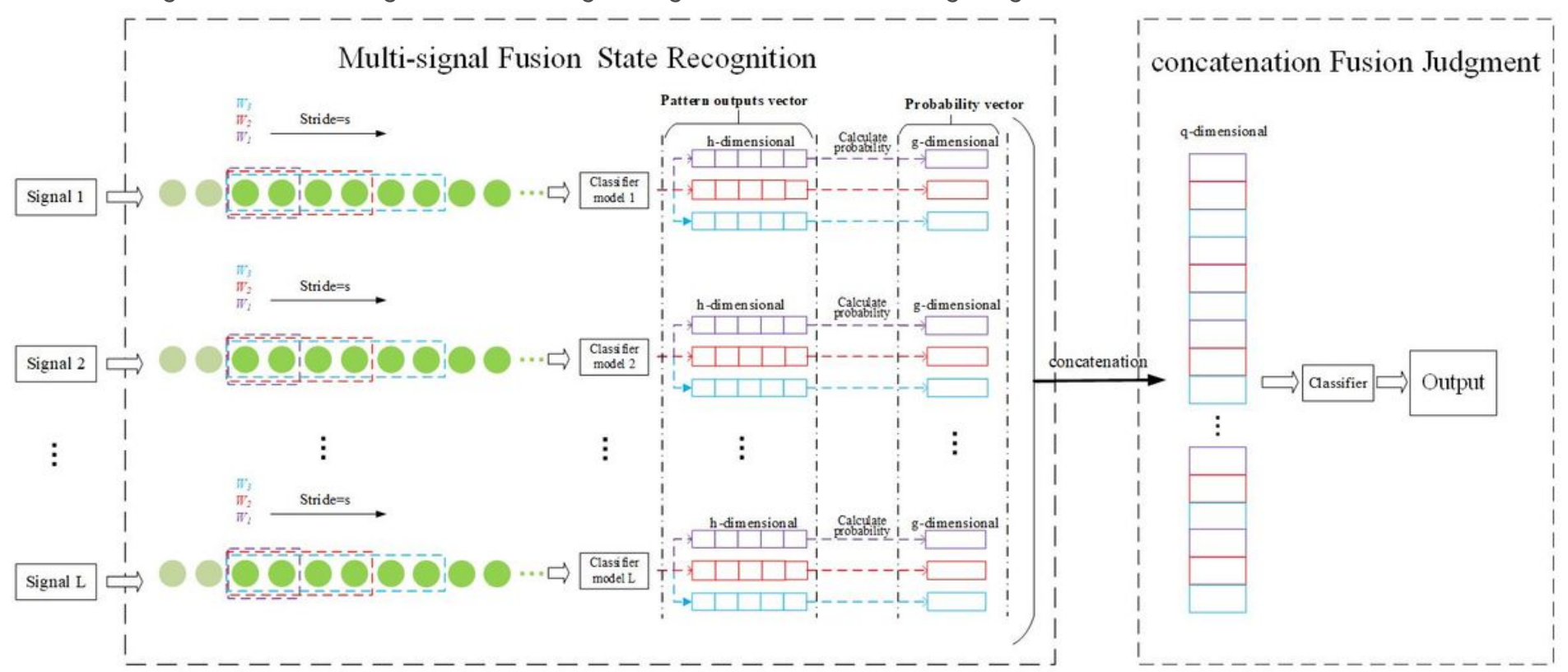

\section{Figure 3}

The multi-source pattern recognition model framework 


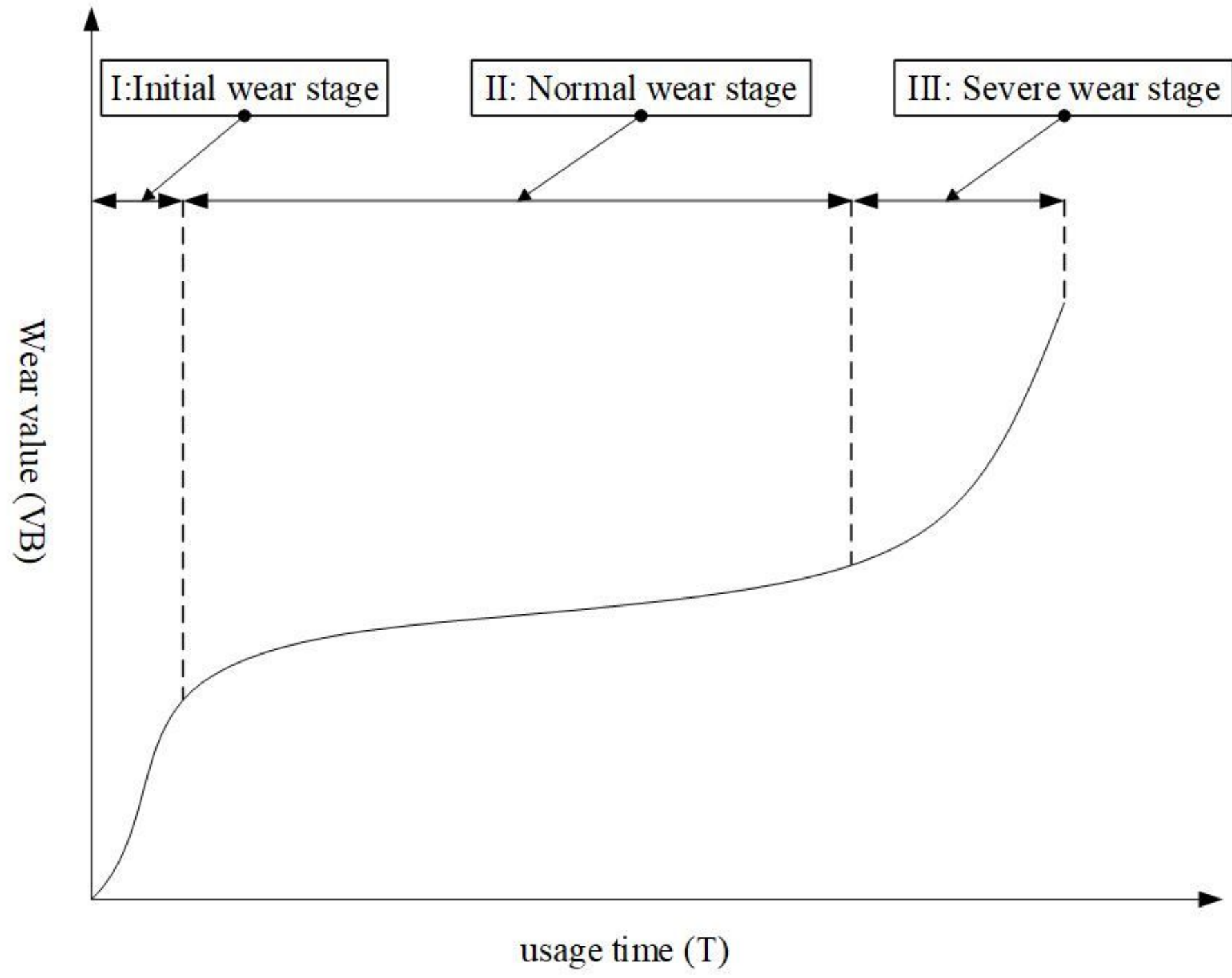

Figure 4

Typical tool wear curve. 


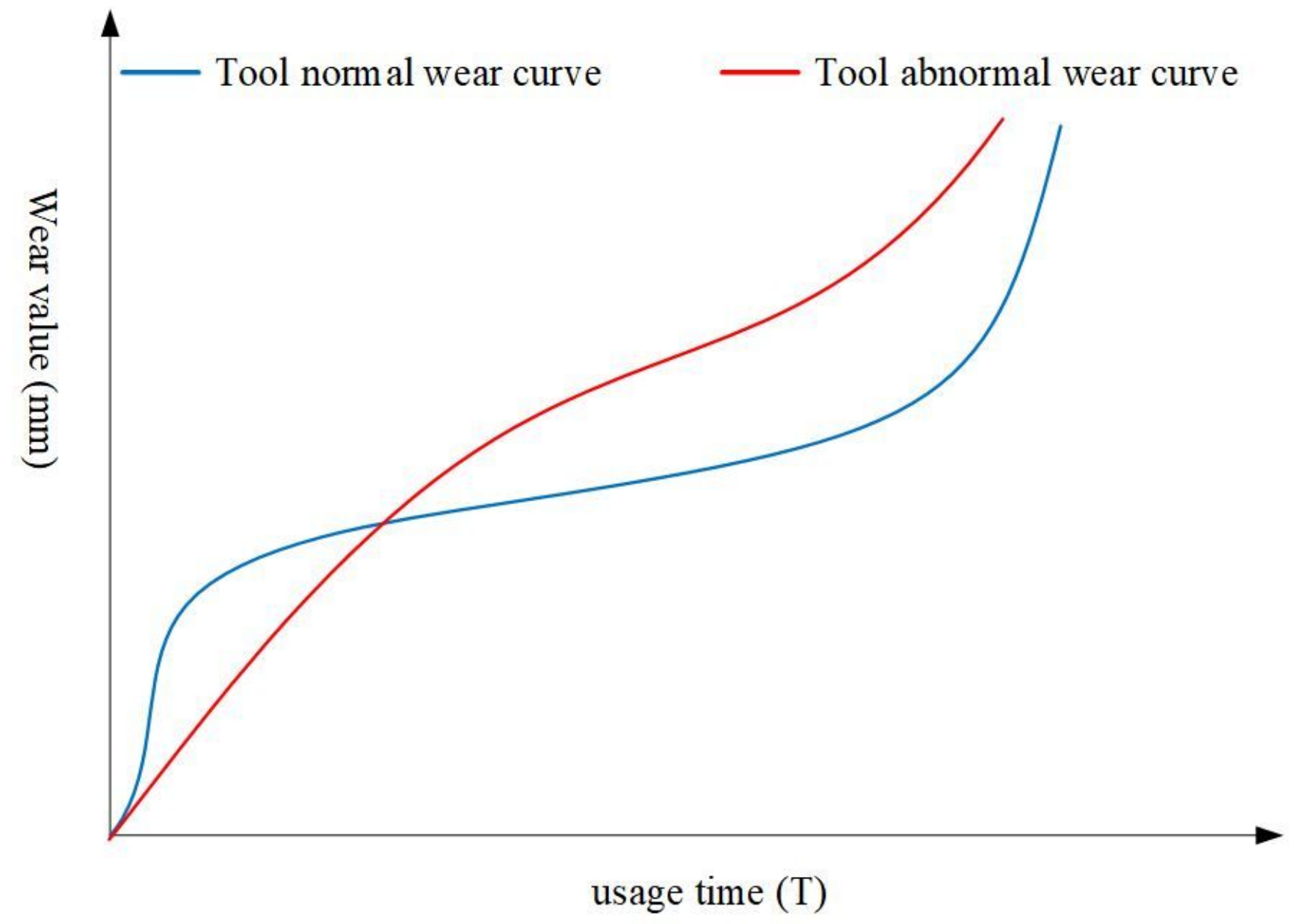

Figure 5

Comparison curve of tool normal wear and abnormal wear. 


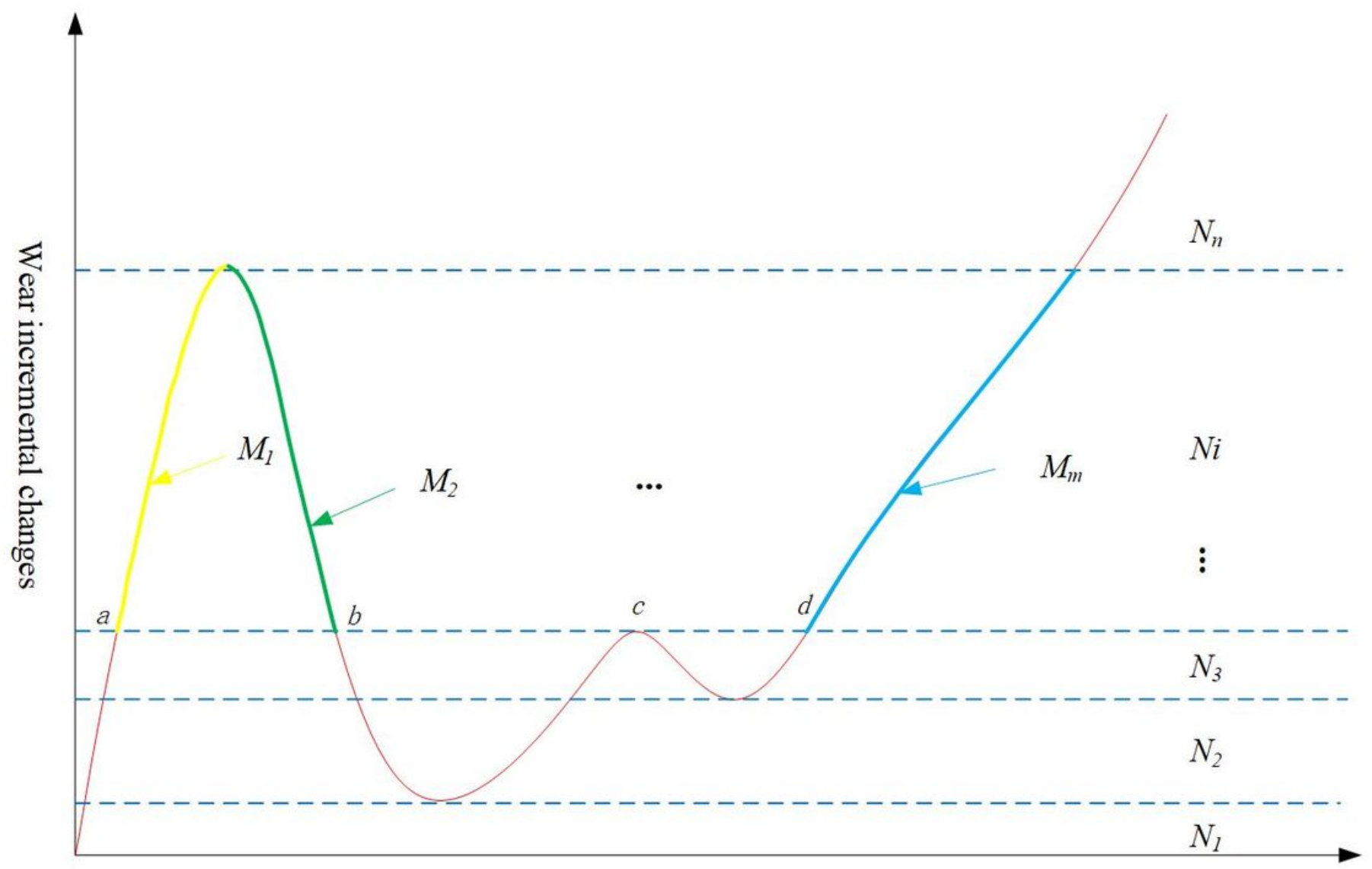

Processing time

\section{Figure 6}

schematic diagram of the change of tool wear increment.

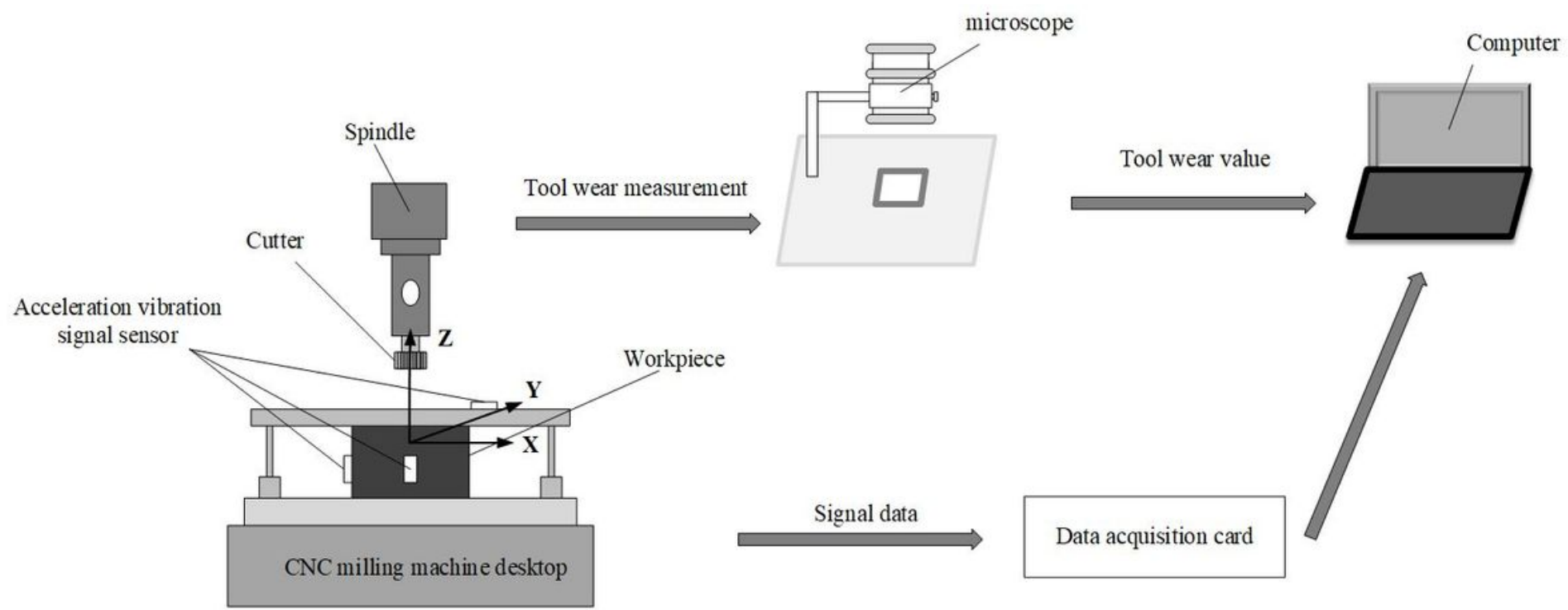

\section{Figure 7}

The experiment structure of the 2010 International PHM Data Challenge Competition test 


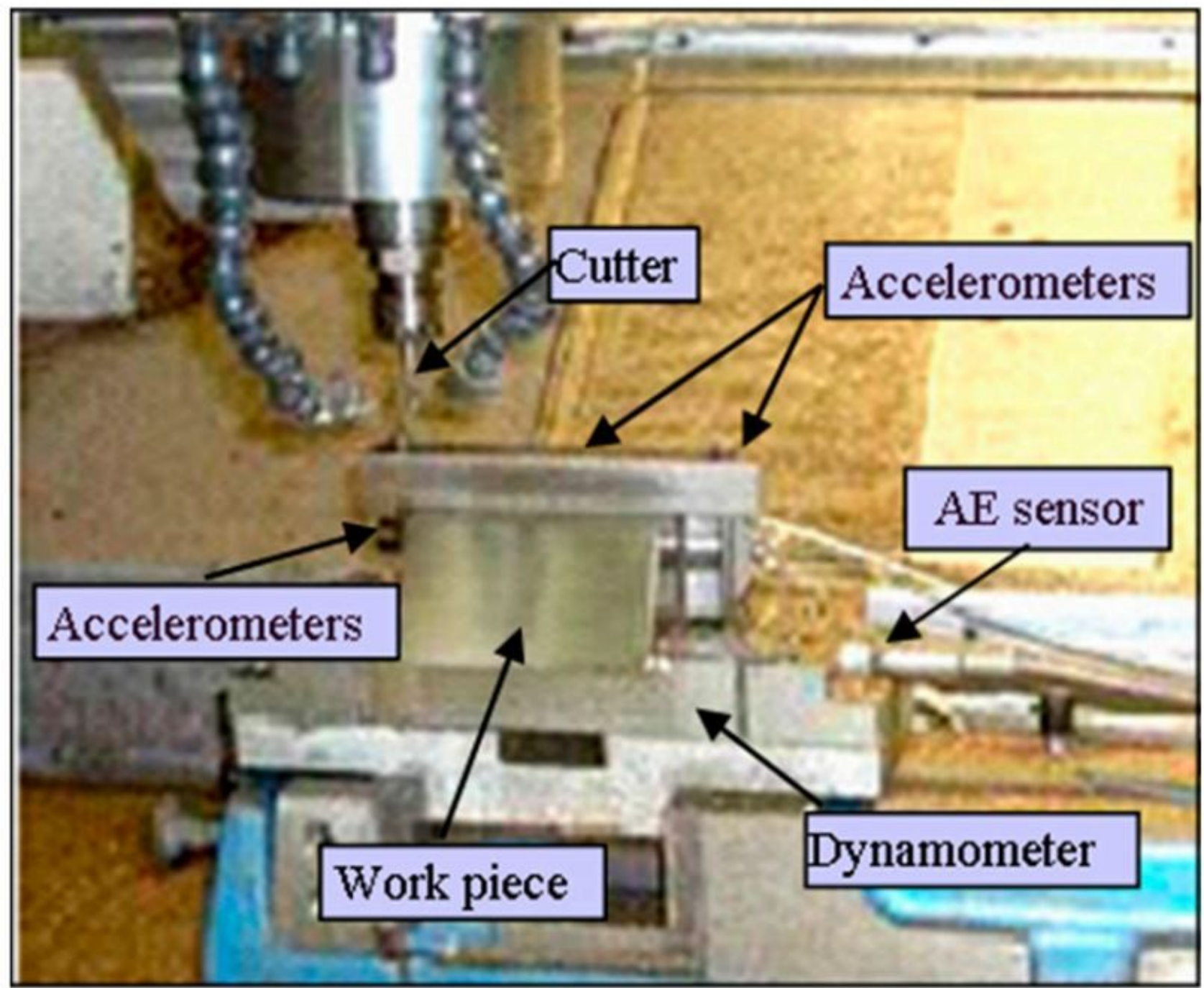

Figure 8

The experimental actual installation 

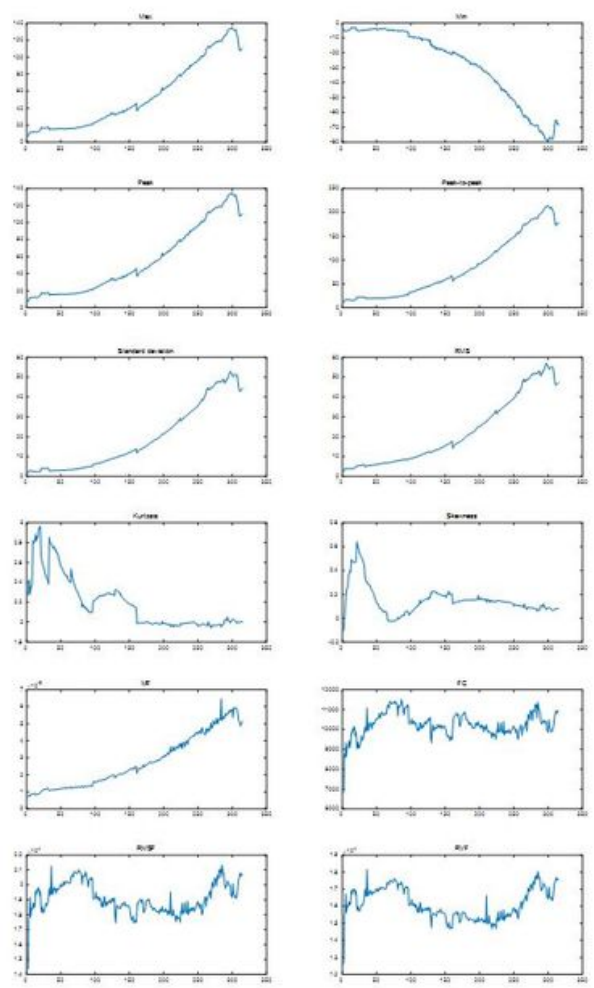
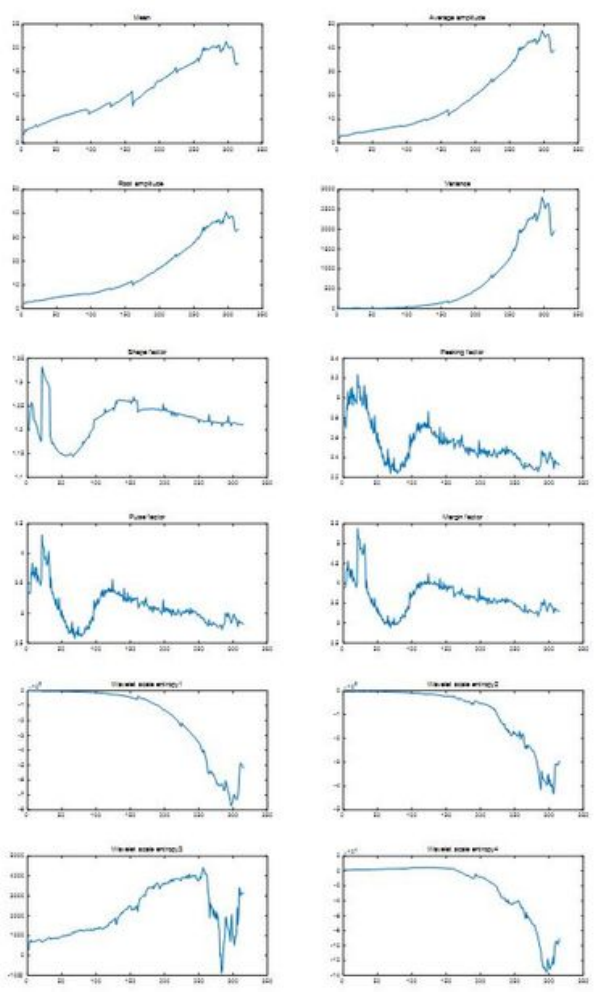
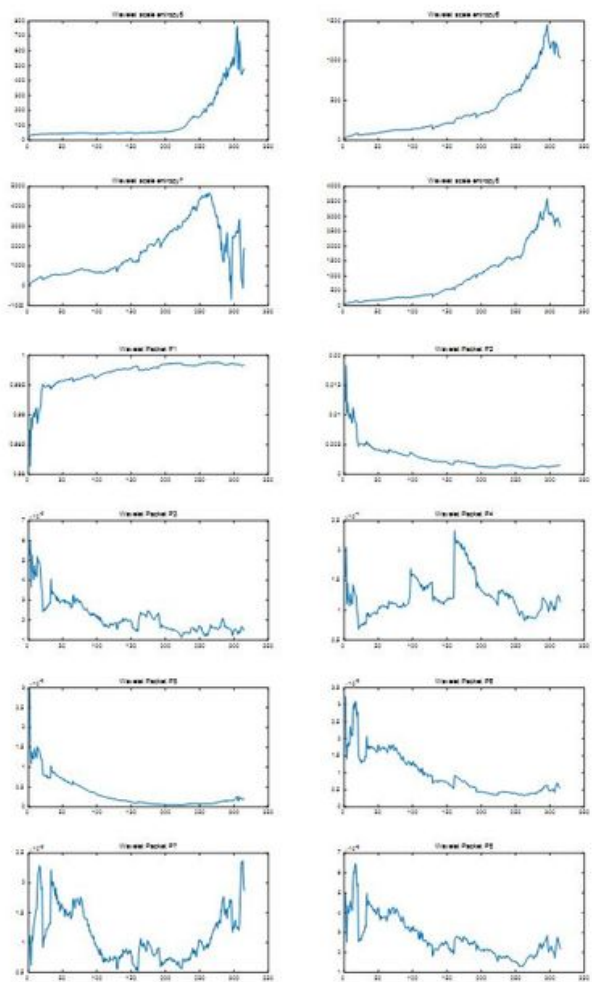
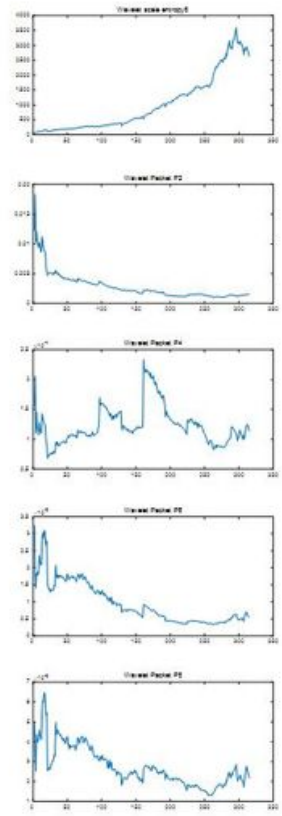

Figure 9

Partial result of signal extraction

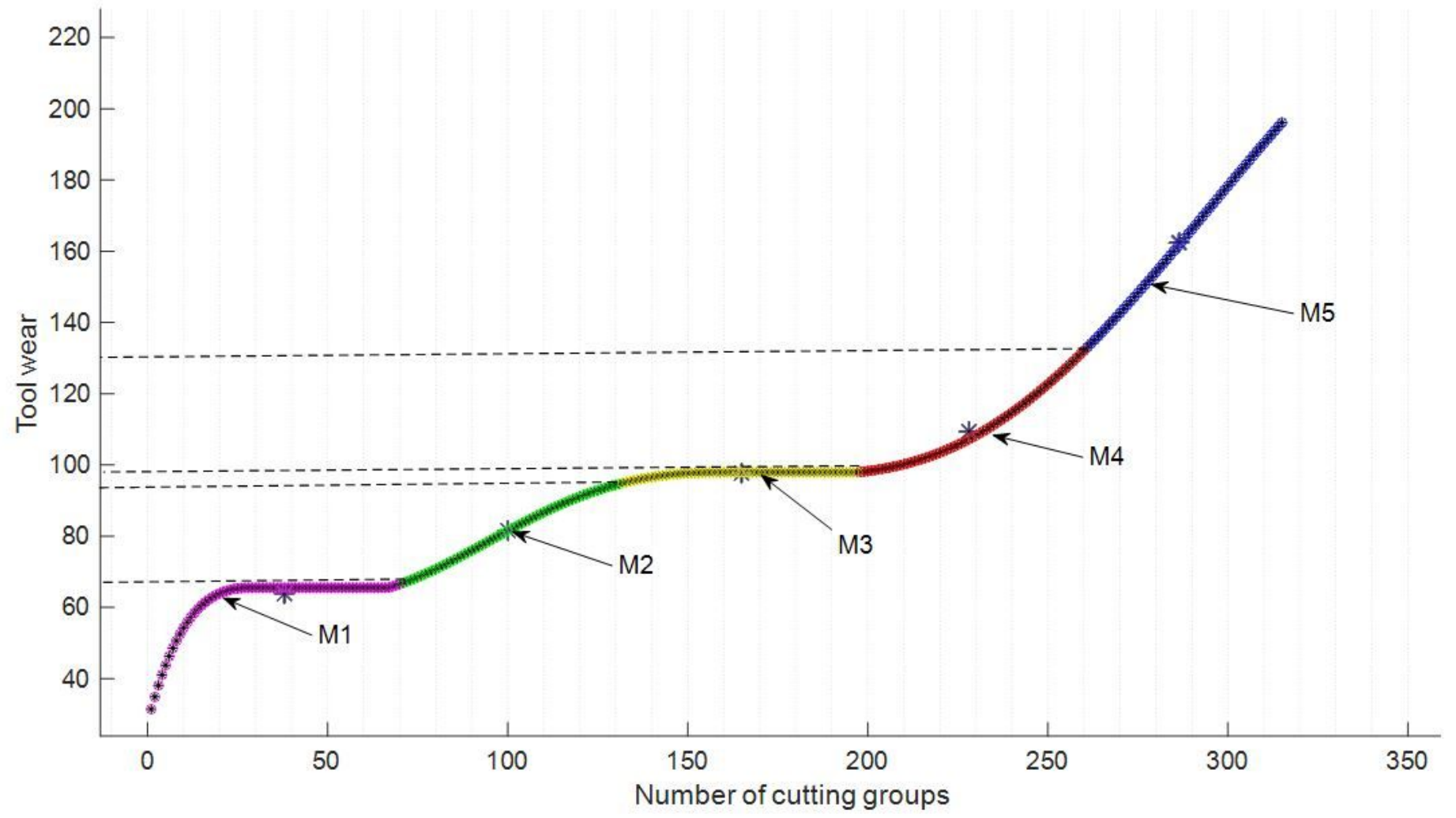

\section{Figure 10}

The result of tool wear pattern division 


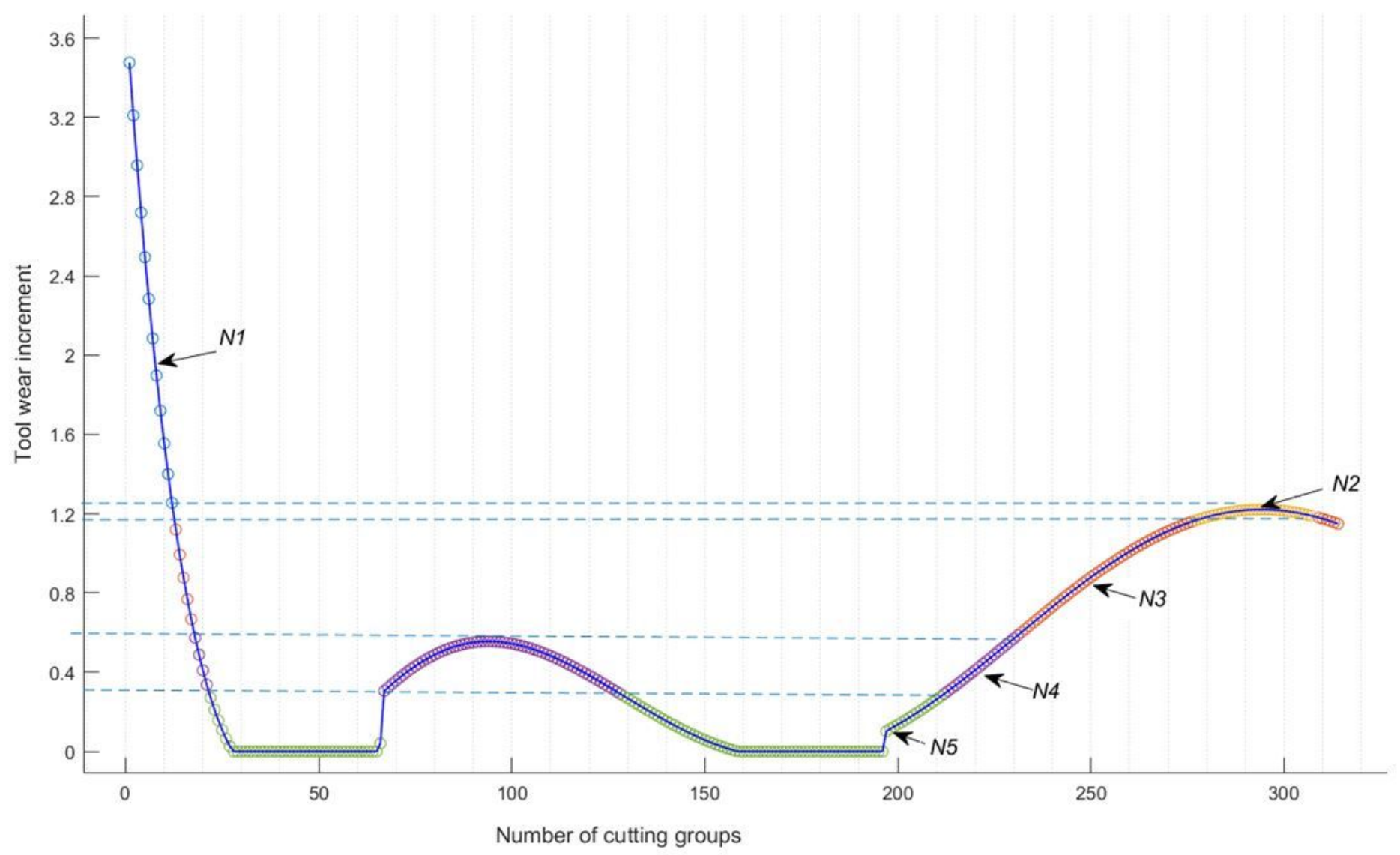

\section{Figure 11}

The result of tool wear increment pattern division

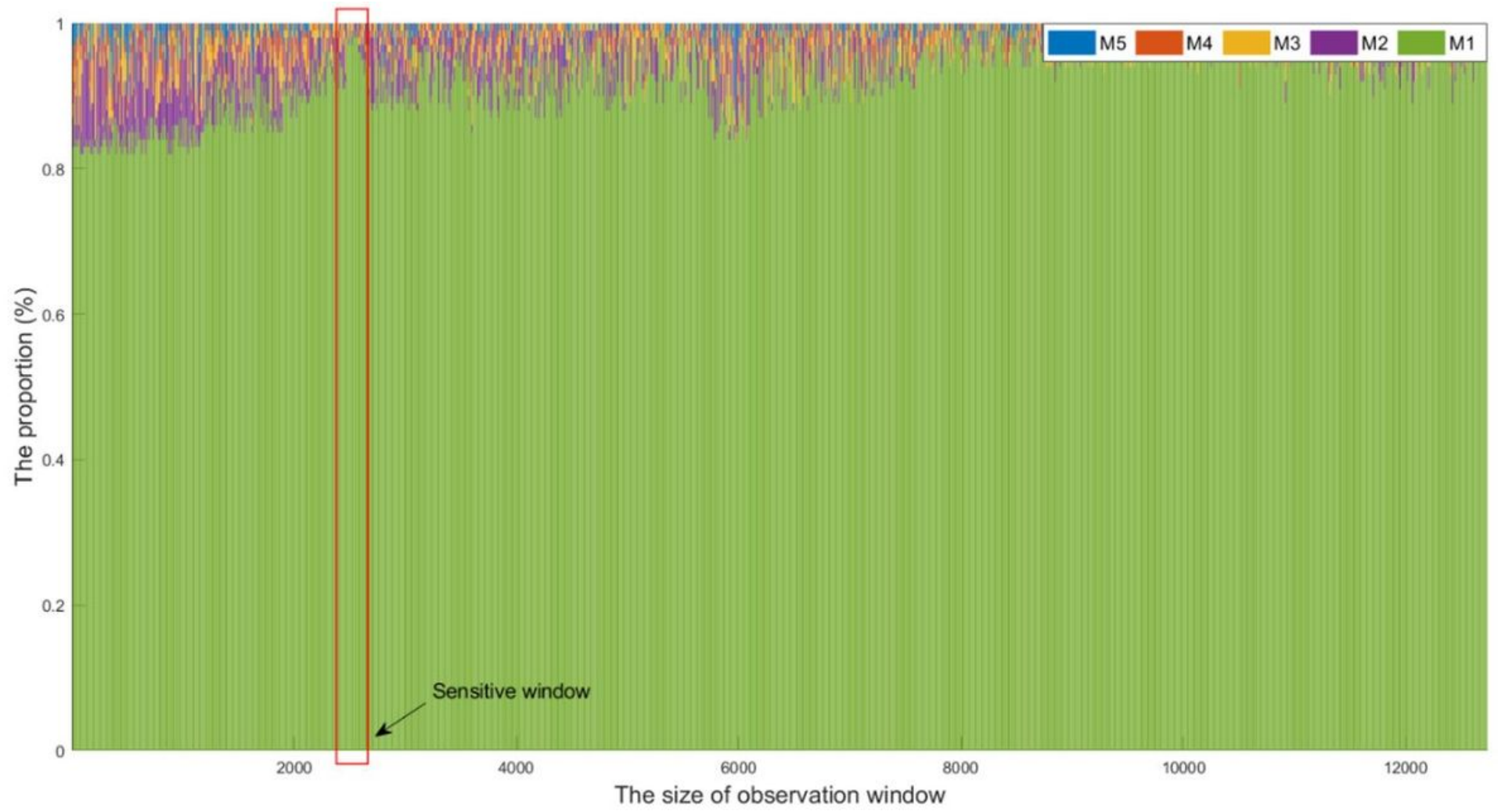

Figure 12

Page 19/22 


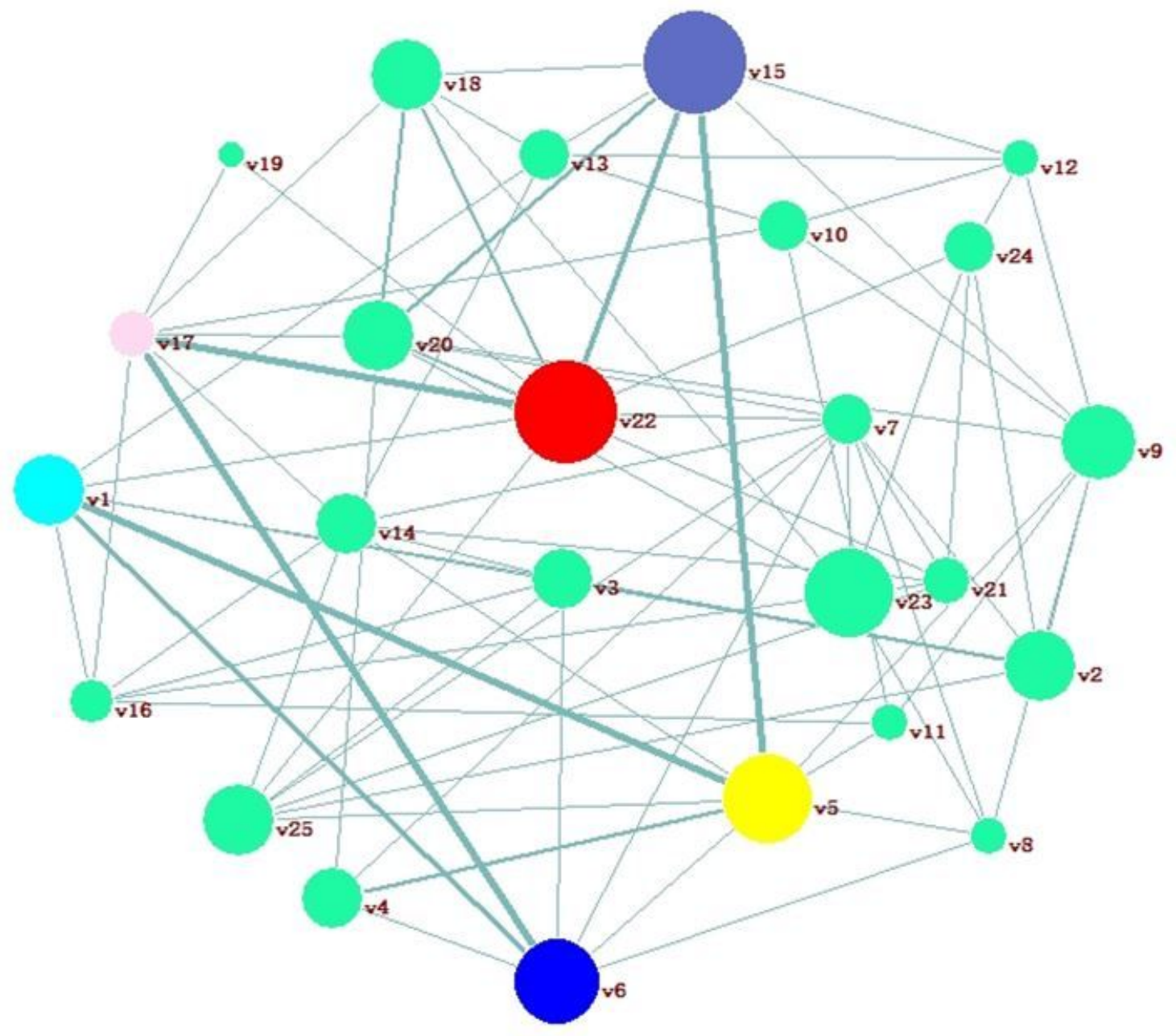

\section{Figure 13}

Tool wear pattern transfer path diagram 


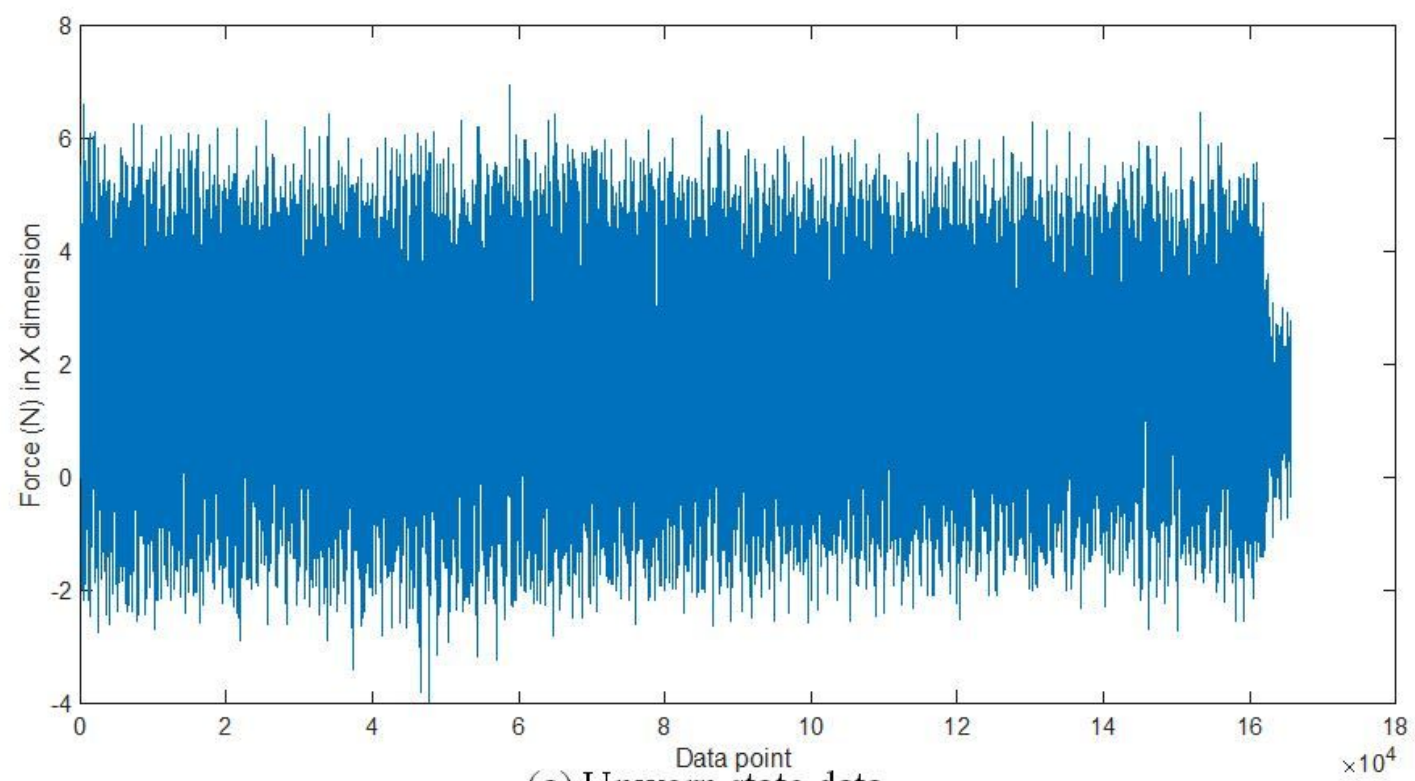

(a) Unworn state data

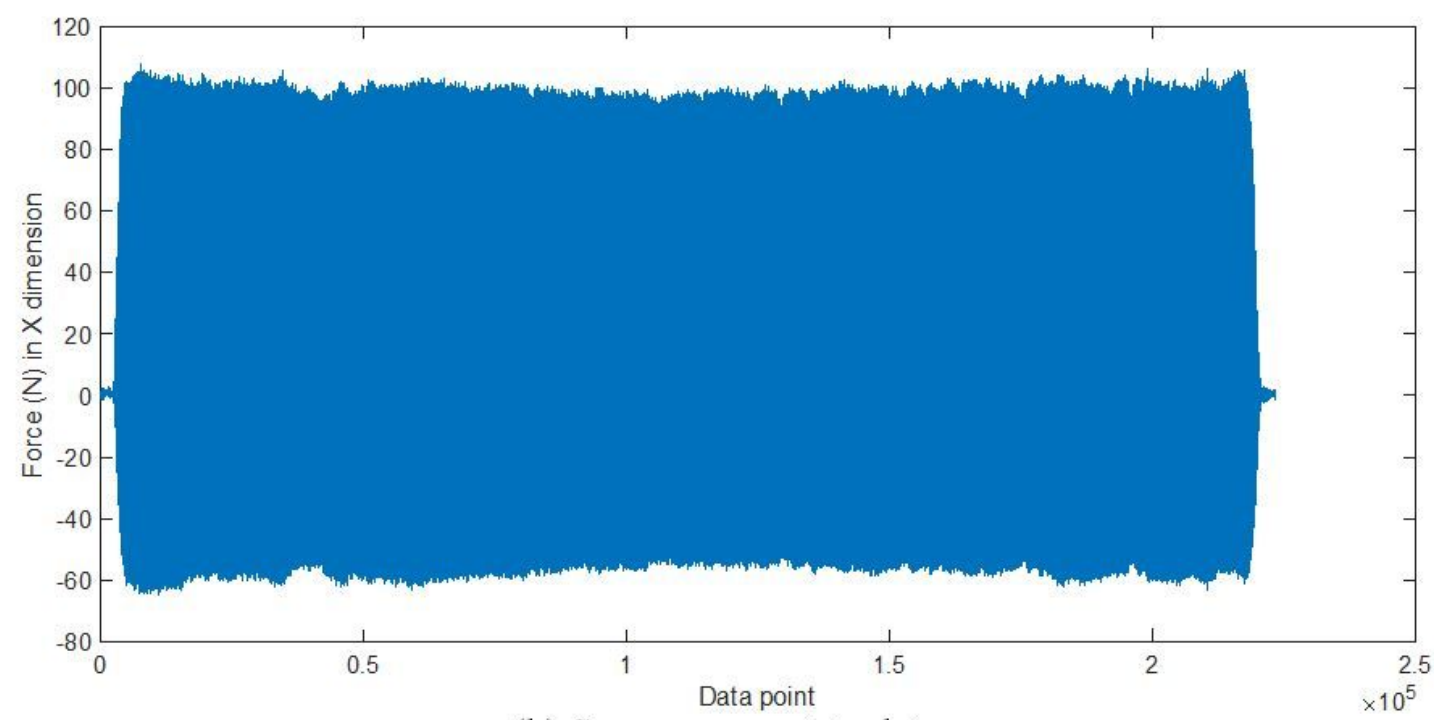

(b) Severe wear state data

Figure 14

The comparison of unworn and severe wear state by cutting force $(\mathrm{N})$ in $\mathrm{X}$ dimension signals 


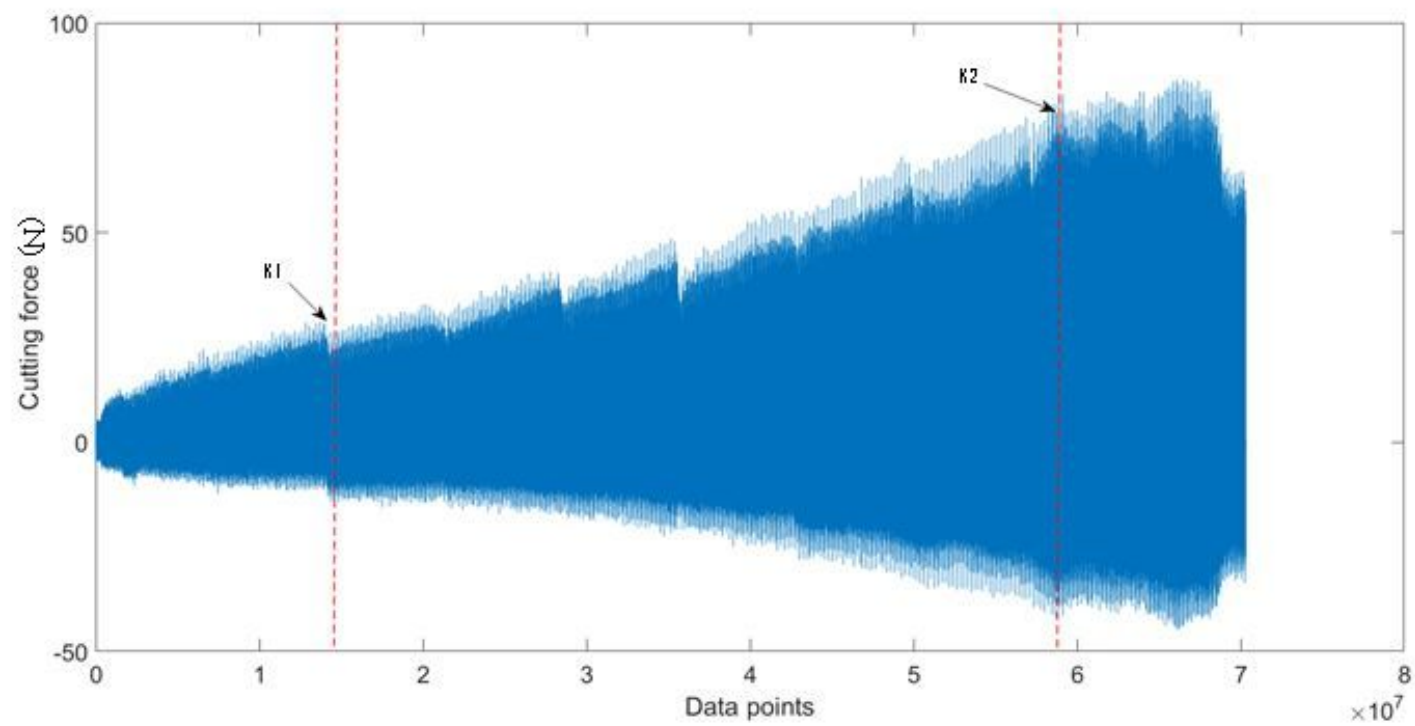

(a) The force signal for monitoring

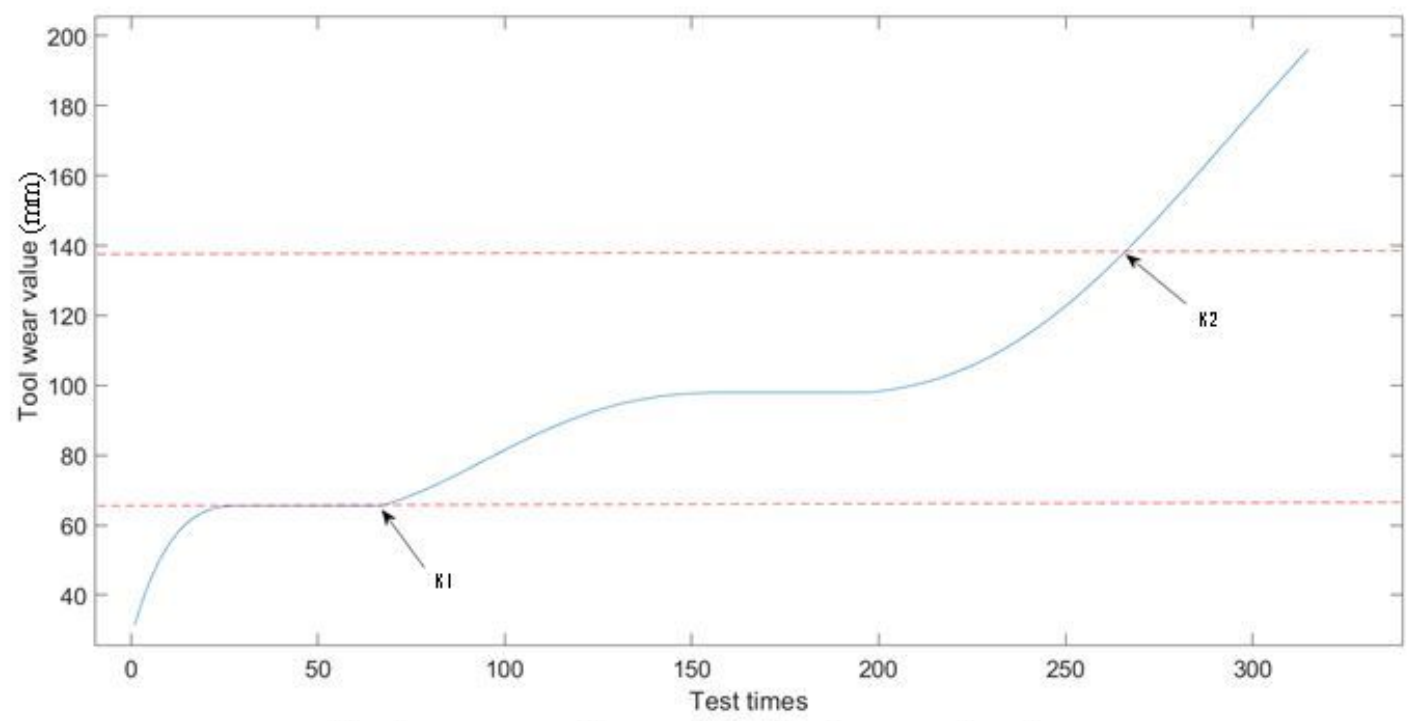

(b) The corresp onding statistical tool wear value change curve

\section{Figure 15}

The result of wear state recognition 